\title{
Generating $\alpha$-dense curves in non-convex sets to solve a class of non-smooth constrained global optimization
}

\author{
Mohamed Rahal $^{1, *}$, Abdelkader Ziadi ${ }^{1}$, Rachid Ellaia ${ }^{2}$ \\ ${ }^{1}$ Laboratory of Fundamental and Numerical Mathematics \\ Department of Mathematics, Ferhat Abbas, Sétif 1 University \\ Sétif 19000, Algeria \\ E-mail:〈mrahaldz@gmail.com,ziadiaek@yahoo.fr〉 \\ ${ }^{2}$ Laboratory of Study and Research for Applied Mathematics \\ Mohammadia School of Engineers, BP. 765, Ibn Sina Avenue \\ Agdal, Rabat, Morocco \\ E-mail:〈rachid.ellaia@gmail.com〉
}

\begin{abstract}
This paper deals with the dimensionality reduction approach to study multi-dimensional constrained global optimization problems where the objective function is non-differentiable over a general compact set $D$ of $\mathbb{R}^{n}$ and Hölderian. The fundamental principle is to provide explicitly a parametric representation $x_{i}=\ell_{i}(t), 1 \leq i \leq n$ of $\alpha$-dense curve $\ell_{\alpha}$ in the compact $D$, for $t$ in an interval $\mathbb{I}$ of $\mathbb{R}$, which allows to convert the initial problem to a one-dimensional Hölder unconstrained one. Thus, we can solve the problem by using an efficient algorithm available in the case of functions depending on a single variable. A relation between the parameter $\alpha$ of the curve $\ell_{\alpha}$ and the accuracy of attaining the optimal solution is given. Some concrete $\alpha$-dense curves in a non-convex feasible region $D$ are constructed. The numerical results show that the proposed approach is efficient.
\end{abstract}

Keywords: $\alpha$-dense curves, constrained global optimization, non-smooth non-convex functions, Hölder condition, Piyavskii's algorithm

Received: November 3, 2018; accepted: October 16, 2019; available online: December 13, 2019

DOI: $10.17535 /$ crorr.2019.0024

\section{Introduction}

Let us consider the following constrained global optimization problem

$$
\left\{\begin{array}{l}
\min F(x) \\
\text { subject to } g_{i}(x) \leq 0, i \in I \\
x \in \Omega,
\end{array}\right.
$$

where $x=\left(x_{1}, \cdots, x_{n}\right)^{T}$ is the real vector of $\mathbb{R}^{n}$ representing the $n$ variables, $I$ is a finite index set and $\Omega$ is a compact hyper-rectangle given by

$$
\Omega=\left[x_{1}^{l}, x_{1}^{u}\right] \times \cdots \times\left[x_{n}^{l}, x_{n}^{u}\right] \subset \mathbb{R}^{n},
$$

and $x_{i}^{l}, x_{i}^{u}, i=1, \cdots, n$ are real constants specifying the hyper-rectangle $\Omega$. The functions $F, g_{i}(i \in I): \Omega \rightarrow \mathbb{R}$ are assumed to be Hölderian and non-differentiable over $\Omega$. Denote the

${ }^{*}$ Corresponding author. 
feasible region of solutions for problem (1) associated to inequality constraints and explicit bounds by

$$
D=\left\{x \in \Omega, g_{i}(x) \leq 0, \quad \forall i \in I\right\},
$$

with the assumption that the feasible region $D$ is bounded and robust, i.e. it is the closure of a non-empty open set.

Definition 1. A function $F: \Omega \rightarrow \mathbb{R}$, is said to be Hölder continuous on a hyper-rectangle $\Omega$, if there exists a pair of finite positive constants $H$ and $\beta<1$, satisfying

$$
|F(x)-F(y)| \leq H\|x-y\|^{\beta}, \quad \forall x, y \in \Omega,
$$

where $\|$.$\| denotes the Euclidean vector norm in \mathbb{R}^{n}$, other norms could be also considered.

The two real constants $H$ and $\beta$ are respectively called Hölder constant and Hölder exponent. If $F$ is a Hölder function with constant $H$ and exponent $\beta$ on $\Omega$, then it is also Hölderian with constant $H^{\prime} \geq H$ or exponent $\beta^{\prime} \leq \beta$. Note that a Hölder function need not be differentiable. A Hölder function with a low value of $\beta$ is much more irregular than a Hölder function with a large value of $\beta$.

Global optimization is a field with active research. It is the process of finding the global extremum of a function of $n$ variables, with the possibility of being subjected to some constraints. Its importance is due to the increasing needs in many applications in sciences and engineering. Two factors render the global optimization of certain classes of multivariate multiextremal functions difficult to treat: local irregularity, for example non differentiability and the existence of a large number of local and global extrema of the objective function in the feasible area. During the last recent years, numerous works have been realized concerning Lipschitz functions $[7,8,15,21]$. More recently, some works tackling less regular functions have appeared $[4,10,12,17]$. The global maximisation of both univariate and multivariate Hölder functions over respectively an interval of $\mathbb{R}$ and a hyper-rectangle of $\mathbb{R}^{n}$ was studied for the first time by Gourdin et al. [4]. In [4], the authors used the generalization of Piyavskii's algorithm to univariate case. For the multivariate case, the direct generalization of the Piyavskii's algorithm is very difficult, since the determination of local maxima of the over-estimator function of the objective function requires the determination of the intersection of several parabolic hyper-surfaces. They proposed a procedure for partitioning and eliminating (Branch-and-Bound) hyper-rectangles without interest by constructing piecewise constant overestimator functions. Lera and Sergeyev [10] proposed another extension of Piyavskii's algorithm in the one-dimensional case, since the determination of the explicit unique local minimum of the under-estimator function is generally easy only for some cases when the Hölder exponent $\beta=\frac{1}{m}$ and $m$ is the integers 2,3 and 4. They also consider a technique if the Hölder constant $h$ is not a priori known.

In [17], we have proposed two algorithms of both univariate and multivariate unconstrained Hölder functions. The first one is a modification of the Piyavskii's algorithm for the univariate case. It consists on constructing a sequence of piecewise affine under-estimators of the objective function. The second algorithm is the reduction of the multivariate Hölder objective function to a univariate one, by using a parameterized curves as later described.

The aim of this paper is to study the extension of the modified Piyavskii's algorithm MPA which we have introduced in [17] for minimizing multivariate Hölderian functions over a hyperrectangle of $\mathbb{R}^{n}[6]$ to minimization problems of strictly Hölder functions of several variables undergoing a finite set of constraints which are also strictly Hölderian.

In many cases, the feasible region is a non-convex compact set whose boundaries are nonsmooth functions. We treat the problem (1) in the case where the feasible set $D$ can be formulated, with a finite number of inequalities, as follows: 


$$
D=\left\{\begin{array}{c}
a \leq x_{1} \leq b \\
x \in \Omega, \quad \\
\\
\varphi_{i}\left(x_{1}, \ldots, x_{i-1}\right) \leq x_{i} \leq \psi_{i}\left(x_{1}, \ldots, x_{i-1}\right), 2 \leq i \leq n
\end{array}\right\},
$$

where $a, b \in \mathbb{R}$ such that $a \leq x_{1}^{l}, b \leq x_{1}^{u}$ and the functions $\varphi_{i}$ and $\psi_{i}(i \geq 2)$ are all Hölderian and non-differentiable over a hyper-rectangles $\Omega_{i}$ given by

$$
\Omega_{i}=\left[x_{1}^{l}, x_{1}^{u}\right] \times \ldots \times\left[x_{i-1}^{l}, x_{i-1}^{u}\right],
$$

respectively with the constants $h_{i}>0, \beta_{i}<1$ and $H_{i}>0, \gamma_{i}<1$, that is,

$$
\begin{aligned}
& \left|\varphi_{i}(x)-\varphi_{i}(y)\right| \leq h_{i}\|x-y\|^{\beta_{i}}, \quad \forall x, y \in \Omega_{i} \\
& \left|\psi_{i}(x)-\psi_{i}(y)\right| \leq H_{i}\|x-y\|^{\gamma_{i}}, \quad \forall x, y \in \Omega_{i} .
\end{aligned}
$$

As the problem (1) is generally difficult to solve, there are only a few works in the literature $[3,13]$. In this paper, we develop an approach which does not use penalty functions or Lagrange multipliers. It is based on the reduction of the dimension of problem (1) where the feasible set $D$ is defined by (3), by means of a transformation allowing to write the $n$ variables $x_{i}, 1 \leq i \leq n$ in functions of a single variable $t$, given by $x_{i}=\ell_{i}(t), 1 \leq i \leq n$. The objective function $F(x)$ of the initial problem (1) is approximated by a function of a single variable defined by $f(t)=F\left(\ell_{1}(t), \ldots, \ell_{n}(t)\right)$. A computable parametric $\alpha$-dense curve $\ell_{\alpha}(t)=\left(\ell_{1}(t), \ldots, \ell_{n}(t)\right)$ in the feasible region $(3)$ should be used and ensured the passage from the constrained multidimensional problem (1) and (3) to the unconstrained one-dimensional simpler one

$$
\min _{t \in \mathbb{I}} f(t),
$$

where the univariate objective function $f(t)$ of the problem (4) is Hölderian and non differentiable over the interval $\mathbb{I}=[0, T]$. The constant $T$ is the upper bound of the function $\ell_{\alpha}(t)$ on which the global minimum of $f(t)$ in $\mathbb{I}$ will be located. It can be proved that the global minimum of the function $F(x)$ on the compact $D$ is approximated by the global minimum of the function $f(t)$ on the interval $\mathbb{I}$. This due to the fact that $f(t)$ is the restriction of $F(x)$ to the $\alpha$-dense curve in $D$. Thus, we can solve the problem (4) by applying a more efficient and performing algorithm proposed for minimizing functions depending on a single variable and satisfying the Hölder condition (2). It should be mentioned that recently, the dimensionality reduction approach, coupled with some one-dimensional global optimization methods has proved its effectiveness in solving various multi-dimensional problems where the objective function is Lipschitzian and the feasible set is a relatively small hyper-rectangle [1, 17, 23, 24].

The paper is organized as follows. In Section 2, we give some preliminaries and notations. The dimensionality reduction method is presented with concrete $\alpha$-dense curves in Section 3. In

Section 4 we give an algorithm for a one-dimensional unconstrained problem. Section 5 shows some numerical experiments and Section 6 concludes the work.

\section{Preliminaries and notation}

\subsection{Lower and upper bounds}

In the preliminaries, we start by recalling that the diameter of the hyper-rectangle $\Omega_{i}$ for each $i=1, \ldots, n$ is

$$
d_{i}=\max \left\{\|x-\widetilde{x}\|, x, \widetilde{x} \in \Omega_{i}\right\}=\sqrt{\left(x_{1}^{l}-x_{1}^{u}\right)^{2}+\ldots+\left(x_{i-1}^{l}-x_{i-1}^{u}\right)^{2}}
$$


and the center of $\Omega_{i}$ is

$$
c_{i}=\frac{1}{2}\left(x_{1}^{l}+x_{1}^{u}, \ldots, x_{i-1}^{l}+x_{i-1}^{u}\right)
$$

Let the following numbers:

$$
\left\{\begin{array}{l}
L_{i}=\varphi_{i}\left(c_{i}\right)-h_{i}\left(\frac{d_{i}}{2}\right)^{\beta_{i}} \\
U_{i}=\psi_{i}\left(c_{i}\right)+H_{i}\left(\frac{d_{i}}{2}\right)^{\gamma_{i}} .
\end{array}\right.
$$

Since the functions $\varphi_{i}$ and $\psi_{i}(i \geq 2)$ are Hölderian on $\Omega_{i}$ then by the Hölder condition (2) we have

$$
\begin{aligned}
\varphi_{i}(y)-h_{i}\|x-y\|^{\beta_{i}} & \leq \varphi_{i}(x), \text { for all } x, y \in \Omega_{i} \\
\psi_{i}(y)-H_{i}\|x-y\|^{\gamma_{i}} & \leq \psi_{i}(x), \text { for all } x, y \in \Omega_{i} .
\end{aligned}
$$

In the last two inequalities, if a value of $y$ is fixed by the center $c_{i}$ of $\Omega_{i}$, then, the constants $L_{i}$ and $U_{i}$ given above, are respectively lower and upper bounds of the functions $\varphi_{i}$ and $\psi_{i}$ on the hyper-rectangle $\Omega_{i}$, for $i \geq 2$, with $L_{1}=a$ and $U_{1}=b$ [4].

\subsection{Notation and sequence of parameters}

In all of this work, the constants $h_{i}, H_{i}, \beta_{i}, \gamma_{i}$, are assumed to be known a priori and selected such $\left(h_{i}\right)_{2 \leq i \leq n},\left(H_{i}\right)_{2 \leq i \leq n}$ form two increasing sequences and $\left(\beta_{i}\right)_{2 \leq i \leq n},\left(\gamma_{i}\right)_{2 \leq i \leq n}$ two decreasing ones. Let $\alpha>0$ and very small compared to the feasible region $D$ and let, for $i \geq 2$,

$$
\left\{\begin{array}{c}
\delta_{1}=1, \delta_{i}=1+\max \left(h_{i} \alpha^{\beta_{i}-1} \delta_{i-1}^{\beta_{i}}, H_{i} \alpha^{\gamma_{i}-1} \delta_{i-1}^{\gamma_{i}}\right) \\
\lambda_{i}=\max \left(1, h_{i}\left(\alpha \delta_{i-1}\right)^{\beta_{i}-1}, H_{i}\left(\alpha \delta_{i-1}\right)^{\gamma_{i}-1}\right) .
\end{array}\right.
$$

The sequence of numbers $\left(\delta_{i}\right)_{1 \leq i \leq n}$ is strictly increasing. Indeed, for $i=2$, since $h_{2}>0$, $\beta_{2}<1$ and $H_{2}>0, \gamma_{2}<1$, then $\delta_{2}>\delta_{1}$. We suppose that $\delta_{i}<\delta_{i+1}$ for all $i$, we have

$$
h_{i+1}<h_{i+2} \text { and } \beta_{i+2}<\beta_{i+1} \text { then for } \alpha<<1 \text { we have } \alpha^{\beta_{i+1}}<\alpha^{\beta_{i+2}} \text {. }
$$

Analogously,

$$
H_{i+1}<H_{i+2} \text { and } \gamma_{i+2}<\gamma_{i+1} \text { then } \alpha^{\gamma_{i+1}}<\alpha^{\gamma_{i+2}} .
$$

By suitably choosing the sequence $\delta_{i}$ such that $\delta_{i}<\delta_{i+1}$ we have

$$
\delta_{i}^{\beta_{i+1}}<\delta_{i+1}^{\beta_{i+2}} \text { and } \delta_{i}^{\gamma_{i+1}}<\delta_{i+1}^{\gamma_{i+2}},
$$

however,

$$
\begin{aligned}
h_{i+1} \alpha^{\beta_{i+1}-1} \delta_{i}^{\beta_{i+1}} & <h_{i+2} \alpha^{\beta_{i+2}-1} \delta_{i+1}^{\beta_{i+2}} \\
\text { and } H_{i+1} \alpha^{\gamma_{i+1}-1} \delta_{i}^{\gamma_{i+1}} & <H_{i+2} \alpha^{\gamma_{i+2}-1} \delta_{i+1}^{\gamma_{i+2}},
\end{aligned}
$$

hence $\delta_{i+1}<\delta_{i+2}$.

\section{The dimensionality reduction method}

In the literature, there exist several methods based on the reduction of the dimension of a multidimensional global optimization problem to an univariate case. The method based on filling the feasible region by a curve was studied by numerous authors, see e.g. Butz [2], Strongin [20] and Sergeyev et al. [19]. They consider the approximation of Peano type curves for this purpose. These curves were first introduced by Peano (1890) then Hilbert (1891) and have 
the property of passing through all points of a hyper-rectangle of $\mathbb{R}^{n}$ and are known as spacefilling-curves [18] The process based on the approximation by Peano type curves to explore the feasible region have been systematically developed and improved by Strongin, Sergeyev and their collaborators $[11,20]$. The monograph [19] treats in detail the theoretical and numerical aspects of this approach. The last works involving this approach have shown that it is competitive in practice and specifically when the feasible set is a hyper-rectangle relatively small of dimension $\leq 4[11,20]$. Sergeyev et al. [11] have solved the global minimization problem when the objective function $F(x), x \in \mathbb{R}^{n}$, fulfills the Lipschitz condition on the hyper-rectangle of $\mathbb{R}^{n}$ using computable approximations of Peano functions to reduce the original problem to an univariate one satisfying the Hölder condition over an interval of $\mathbb{R}$. They also proposed some techniques for estimating locally and globally the Lipschitz and the Hölder constants when these constants are not a priori known [11].

The dimensionality reduction approach (DR) that we will present in our work, is different with the approach given above. It has been proposed and developed by Cherruault et al. [1, 5 , $24,26]$ and based on the approximation of an $n$ variables of the objective function by a single variable function by using a continuous parametric curve $\ell_{\alpha}$, defined from a real interval $\mathbb{I}$ onto the $n$-dimensional compact $D$ and satisfying a property described by the definition given below. Such a function $\ell_{\alpha}$ is said to be a space-densifying-curve in $D[23,25]$. However, the introduction of these $\alpha$-dense curves has allowed analytical argumentation of certain dimensionality reduction methods in global optimization techniques [5, 17, 24, 26, 27].

Definition 2. Let $S$ be a subset of the metric space $E$ and $\alpha>0$. We say that a continuous curve $\ell_{\alpha}$ of $E$ is $\alpha$-dense in $S$, if $\ell_{\alpha} \subset S$ and $\mathbf{d}\left(x, \ell_{\alpha}\right) \leq \alpha$ for all $x \in S$, where:

$$
\mathbf{d}\left(x, \ell_{\alpha}\right)=\inf _{y \in \ell_{\alpha}} \mathbf{d}(x, y) \text {. }
$$

Definition 3. Let $\alpha>0$. We say that a subset $S$ of the metric space $E$ is $\alpha$-densifiable if there exists a continuous curve of $E$, rectifiable and $\alpha$-dense in $S$.

Remark 1. Designate by $\mathcal{L}_{\alpha, \ell}$ the length of a continuous curve $\ell_{\alpha}$ parametrically represented by the function $\ell_{\alpha}(t), t \in \mathbb{I}$, where $\mathbb{I}$ is an interval of $\mathbb{R}$. $\left(\mathcal{L}_{\alpha, \ell}\right.$ is independent of the chosen parametrization). We say that the curve $\ell_{\alpha}$ is rectifiable if $\mathcal{L}_{\alpha, \ell}$ is finite.

The curves used in the dimensionality reduction method belong to two different classes. Butz, Strongin and Sergeyev et al. [2, 19, 20], use numerical approximations of Peano type curves whereas Cherruault and their collaborators [24], use $\alpha$-dense ones. The approximations of Peano curves are obviously $\alpha$-dense but the curves used by Cherruault et al. [25] do not necessarily converge to Peano type curves. Although both classes of curves have the common property to approach as much as wanted all the points of a hyper-rectangle of $\mathbb{R}^{n}$, there are important differences: they do not possess the same regularity, they differ also concerning repartition uniformity (on the hyper-rectangle) and asymptotic behavior. In this work, we are interested by compact sets of type (3) which are $\alpha$-densifiable and note that if the functions $\varphi_{i}$ and $\psi_{i}$ are only Hölderian we can construct compacts which are not $\alpha$-densifiable, in this case we can transform our problem by defining "a Lagrange penalty function" by choosing a huge hyper-rectangle $C \subset \mathbb{R}^{n}$ which comprises the feasible set of the problem (1) and so we solve the transformed problem where the objective function is Hölderian on the hyper-rectangle $C$ [17].

\subsection{Generation of $\alpha$-dense curves in a compact non-convex sets}

Some interesting results concerning the existence of $\alpha$-dense curves with minimal length of an $\alpha$-densifiable compact in a general metric space were given by Ziadi et al. [25]. In the case where the feasible set is a hyper-rectangle $\Omega$ of $\mathbb{R}^{n}$, some ways of constructing the $\alpha$-dense curves in $\Omega$ have been given [14, 24, 26,27]. In this subsection we shall give the relationship existing between the components $\ell_{i}(i=1, \ldots, n)$ of the parametrization in order to generate a 
new class of $\alpha$-dense curves in a compact $D \subset \mathbb{R}^{n}$ whose boundary is defined by a non-smooth functions. Denote by $\mathbf{m}$ the Lebesgue measure, the number $\alpha>0$ is supposed to be less than the length of the intervals $\left[\ell_{i}\left(t_{i}^{\prime}\right), \ell_{i}\left(t_{i}^{\prime \prime}\right)\right], t_{i}^{\prime}, t_{i}^{\prime \prime} \in \mathbb{I} \quad \forall i=2, \ldots, n$.

In the rest of this section, the sup-norm $\|x\|:=\max _{1 \leq i \leq n}\left\{\left|x_{i}\right|\right\}$ is used in order to simplify calculations, but other norms can also be considered. Next, keeping the same notations introduced above, we give a general main result for generating $\alpha$-dense curves in $D$.

Theorem 1. Let $\ell_{\alpha}=\left(\ell_{1}, \ldots, \ell_{n}\right): \mathbb{I} \rightarrow D$ be a continuous function and $t_{2}, \ldots, t_{n}, \alpha$ strictly positive numbers such that:

1) $\ell_{1}$ is surjective.

2) For all $i=2, \ldots, n$, and for all interval $\mathbb{I}_{i-1}$ with length $t_{i}$, there exist $t_{i}^{\prime}$ and $t_{i}^{\prime \prime} \in \mathbb{I}_{i-1}$ such that:

$$
\left\{\begin{array}{l}
\ell_{i}\left(t_{i}^{\prime}\right)=\varphi_{i}\left(\ell_{1}\left(t_{i}^{\prime}\right), \ldots, \ell_{i-1}\left(t_{i}^{\prime}\right)\right) \\
\ell_{i}\left(t_{i}^{\prime \prime}\right)=\psi_{i}\left(\ell_{1}\left(t_{i}^{\prime \prime}\right), \ldots, \ell_{i-1}\left(t_{i}^{\prime \prime}\right)\right)
\end{array}\right.
$$

3) For all $i=2, . ., n$, and for all closed interval $\mathbb{J}$, we have:

$$
\mathbf{m}(\mathbb{J})<t_{i} \Rightarrow \mathbf{m}\left(\ell_{i-1}(\mathbb{J})\right)<\alpha .
$$

Then, the parameterized curve defined by $\ell_{\alpha}(t)=\left(\ell_{1}(t), \ldots, \ell_{n}(t)\right)$ for $t \in \mathbb{I}$, is $\delta_{n-1} \lambda_{n} \alpha$ dense in $D$ where $\delta_{n-1}, \lambda_{n}$ are the parameters designated in section 2.

Proof. The proof is obtained by induction in two parts.

Part 1. $n=2$.

Consider the interval $\left[x_{1}-\alpha, x_{1}+\alpha\right]$ where $\left(x_{1}, x_{2}\right) \in D$. Since the function $\ell_{1}$ is surjective, there exists a closed interval $\mathbb{I}_{1} \subset \mathbb{I}$ such that:

$$
\ell_{1}\left(\mathbb{I}_{1}\right)=\left[x_{1}-\alpha, x_{1}+\alpha\right] \cap[a, b] .
$$

But $\mathbf{m}\left(\ell_{1}\left(\mathbb{I}_{1}\right)\right) \geq \alpha$, so $\mathbf{m}\left(\mathbb{I}_{1}\right) \geq t_{2}$ Thus, we have for all $t \in \mathbb{I}_{1}:\left|x_{1}-\ell_{1}(t)\right| \leq \alpha$ moreover, there exists $t_{2}^{\prime}, t_{2}^{\prime \prime} \in \mathbb{I}_{1}$ such that:

$$
\left\{\begin{array}{l}
\ell_{2}\left(t_{2}^{\prime}\right)=\varphi_{2}\left(\ell_{1}\left(t_{2}^{\prime}\right)\right) \\
\ell_{2}\left(t_{2}^{\prime \prime}\right)=\psi_{2}\left(\ell_{1}\left(t_{2}^{\prime \prime}\right)\right)
\end{array}\right.
$$

On the other hand, according to the definition of the feasible region $D$, we have:

$$
\varphi_{2}\left(x_{1}\right) \leq x_{2} \leq \psi_{2}\left(x_{1}\right) .
$$

As the functions $\varphi_{2}, \psi_{2}$ are Hölderian on $\Omega_{2}$, respectively with the constants $h_{2}, \beta_{2}, H_{2}, \gamma_{2}$, i.e.,

$$
\left\{\begin{array}{l}
\left|\varphi_{2}\left(x_{1}\right)-\varphi_{2}\left(\ell_{1}\left(t_{2}^{\prime}\right)\right)\right| \leq h_{2}\left|x_{1}-\ell_{1}\left(t_{2}^{\prime}\right)\right|^{\beta_{2}} \leq h_{2} \alpha^{\beta_{2}} \\
\psi_{2}\left(x_{1}\right)-\psi_{2}\left(\ell_{1}\left(t_{2}^{\prime \prime}\right)\right)\left|\leq H_{2}\right| x_{1}-\left.\ell_{1}\left(t_{2}^{\prime \prime}\right)\right|^{\gamma_{2}} \leq H_{2} \alpha^{\gamma_{2}},
\end{array}\right.
$$

then

$$
\ell_{2}\left(t_{2}^{\prime}\right)-h_{2} \alpha^{\beta_{2}} \leq x_{2} \leq \ell_{2}\left(t_{2}^{\prime \prime}\right)+H_{2} \alpha^{\gamma_{2}}
$$

We will study the following three cases:

$$
\left\{\begin{array}{l}
(1) \ell_{2}\left(t_{2}^{\prime}\right)-h_{2} \alpha^{\beta_{2}} \leq x_{2}<\ell_{2}\left(t_{2}^{\prime}\right) \\
(2) \ell_{2}\left(t_{2}^{\prime}\right) \leq x_{2} \leq \ell_{2}\left(t_{2}^{\prime \prime}\right) \\
(3) \ell_{2}\left(t_{2}^{\prime \prime}\right)<x_{2} \leq \ell_{2}\left(t_{2}^{\prime \prime}\right)+H_{2} \alpha^{\gamma_{2}} .
\end{array}\right.
$$


If case (1) holds, we have:

$$
\left|x_{2}-\ell_{2}\left(t_{2}^{\prime}\right)\right| \leq h_{2} \alpha^{\beta_{2}}
$$

and therefore

$$
\left\|x-\ell_{\alpha}\left(t_{2}^{\prime}\right)\right\|=\max \left\{\left|x_{1}-\ell_{1}\left(t_{2}^{\prime}\right)\right|,\left|x_{2}-\ell_{2}\left(t_{2}^{\prime}\right)\right|\right\} \leq \max \left\{\alpha, h_{2} \alpha^{\beta_{2}}\right\} .
$$

If case $(2)$ is satisfied, there exists $t_{0} \in\left[t_{2}^{\prime}, t_{2}^{\prime \prime}\right]$ such that:

$$
x_{2}=\ell_{2}\left(t_{0}\right)
$$

leading to

$$
\left\|x-\ell_{\alpha}\left(t_{0}\right)\right\| \leq \alpha
$$

If the last case (3) holds, we have:

$$
\left|x_{2}-\ell_{2}\left(t_{2}^{\prime \prime}\right)\right| \leq H_{2} \alpha^{\gamma_{2}}
$$

and therefore

$$
\left\|x-\ell_{\alpha}\left(t_{2}^{\prime \prime}\right)\right\| \leq \max \left\{\alpha, H_{2} \alpha^{\gamma_{2}}\right\}
$$

We deduce that the parameterized curve $\ell_{\alpha}(t)=\left(\ell_{1}(t), \ell_{2}(t)\right)$ for $t \in \mathbb{I}$, is $\lambda_{2} \alpha$-dense in $D$ with $\lambda_{2}=\max \left(1, h_{2} \alpha^{\beta_{2}-1}, H_{2} \alpha^{\gamma_{2}-1}\right)$.

Part 2. For $n>2$.

Let $x=\left(x_{1}, \ldots, x_{n}\right) \in D$ and consider the interval $\left[x_{1}-\alpha, x_{1}+\alpha\right]$, since $\ell_{1}$ is surjective, there exists a closed interval $\mathbb{I}_{1} \subset \mathbb{I}$ such that:

$$
\ell_{1}\left(\mathbb{I}_{1}\right)=\left[x_{1}-\alpha, x_{1}+\alpha\right] \cap[a, b]
$$

But $\mathbf{m}\left(\ell_{1}\left(\mathbb{I}_{1}\right)\right) \underset{\prime \prime}{\geq}$, then $\mathbf{m}\left(\mathbb{I}_{1}\right) \geq t_{2}$. It follows that $\left|x_{1}-\ell_{1}(t)\right| \leq \alpha$ for all $t \in \mathbb{I}_{1}$. In addition, there exist $t_{2}^{\prime}, t_{2}^{\prime \prime} \in \mathbb{I}_{1}$ such that:

$$
\left\{\begin{array}{l}
\ell_{2}\left(t_{2}^{\prime}\right)=\varphi_{2}\left(\ell_{1}\left(t_{2}^{\prime}\right)\right) \\
\ell_{2}\left(t_{2}^{\prime \prime}\right)=\psi_{2}\left(\ell_{1}\left(t_{2}^{\prime \prime}\right)\right) .
\end{array}\right.
$$

Since $\left|\ell_{2}\left(t_{2}^{\prime}\right)-\ell_{2}\left(t_{2}^{\prime \prime}\right)\right| \geq \alpha$, then,

$$
\mathbf{m}\left(\ell_{2}\left(\left[t_{2}^{\prime}, t_{2}^{\prime \prime}\right]\right)\right) \geq \alpha
$$

and hence

$$
\mathbf{m}\left(\left[t_{2}^{\prime}, t_{2}^{\prime \prime}\right]\right) \geq t_{3}
$$

On the other hand, we have $\varphi_{2}\left(x_{1}\right) \leq x_{2} \leq \psi_{2}\left(x_{1}\right)$, and as $\varphi_{2}$ and $\psi_{2}$ are Hölderian on $\Omega_{2}$, respectively with the constants $h_{2}, \beta_{2}, H_{2}, \gamma_{2}$, then

$$
\ell_{2}\left(t_{2}^{\prime}\right)-h_{2} \alpha^{\beta_{2}} \leq x_{2} \leq \ell_{2}\left(t_{2}^{\prime \prime}\right)+H_{2} \alpha^{\gamma_{2}} .
$$

We still have to study the three cases:

$$
\left\{\begin{array}{l}
(1) \ell_{2}\left(t_{2}^{\prime}\right)-h_{2} \alpha^{\beta_{2}} \leq x_{2} \leq \ell_{2}\left(t_{2}^{\prime}\right) \\
(2) \ell_{2}\left(t_{2}^{\prime}\right) \leq x_{2} \leq \ell_{2}\left(t_{2}^{\prime \prime}\right) \\
(3) \ell_{2}\left(t_{2}^{\prime \prime}\right) \leq x_{2} \leq \ell_{2}\left(t_{2}^{\prime \prime}\right)+H_{2} \alpha^{\gamma_{2}}
\end{array}\right.
$$


If (1) holds, we take $\mathbb{I}_{2}=\left[t_{2}^{\prime}, t_{2}^{\prime}+t_{3}\right]$.

If (2) is valid, there exists $\mathbb{I}_{2} \subset\left[t_{2}^{\prime}, t_{2}^{\prime \prime}\right]$ such that:

$$
\ell_{2}\left(\mathbb{I}_{2}\right)=\left[x_{2}-\alpha, x_{2}+\alpha\right] \cap\left[\ell_{2}\left(t_{2}^{\prime}\right), \ell_{2}\left(t_{2}^{\prime \prime}\right)\right] .
$$

If the case (3) holds, we take $\mathbb{I}_{2}=\left[t_{2}^{\prime \prime}-t_{3}, t_{2}^{\prime \prime}\right]$.

It is clear that $\mathbb{I}_{2} \subset\left[t_{2}^{\prime}, t_{2}^{\prime \prime}\right]$ and $\mathbf{m}\left(\mathbb{I}_{2}\right) \geq t_{3}$ in the three cases. Indeed, for (2) we have:

$$
\mathbf{m}\left(\ell_{2}\left(\mathbb{I}_{2}\right)\right) \geq \alpha
$$

and hence $\mathbf{m}\left(\mathbb{I}_{2}\right) \geq t_{3}$ from the assumption 3) of Theorem 1 .

In addition, for all $t \in \mathbb{I}_{2}$, we have:

If (1) holds, then

$$
\left|x_{2}-\ell_{2}(t)\right| \leq\left|x_{2}-\ell_{2}\left(t_{2}^{\prime}\right)\right|+\left|\ell_{2}\left(t_{2}^{\prime}\right)-\ell_{2}(t)\right| \leq \alpha+h_{2} \alpha^{\beta_{2}} .
$$

If (2) holds, then

$$
\left|x_{2}-\ell_{2}(t)\right| \leq \alpha .
$$

If the condition (3) holds, then

$$
\left|x_{2}-\ell_{2}(t)\right| \leq\left|x_{2}-\ell_{2}\left(t_{2}^{\prime \prime}\right)\right|+\left|\ell_{2}\left(t_{2}^{\prime \prime}\right)-\ell_{2}(t)\right| \leq \alpha+H_{2} \alpha^{\gamma_{2}} .
$$

Consequently, for all $t \in \mathbb{I}_{2}$, we have:

$$
\left|x_{2}-\ell_{2}(t)\right| \leq \alpha\left(1+\max \left(h_{2} \alpha^{\beta_{2}-1}, H_{2} \alpha^{\gamma_{2}-1}\right)\right)=\alpha \delta_{2} .
$$

We have $\mathbf{m}\left(\mathbb{I}_{2}\right) \geq t_{3}$, then there exist $t_{3}^{\prime}, t_{3}^{\prime \prime} \in \mathbb{I}_{2}$ such that:

$$
\left\{\begin{array}{l}
\ell\left(t_{3}^{\prime}\right)=\varphi_{3}\left(\ell_{1}\left(t_{3}^{\prime}\right), \ell_{2}\left(t_{3}^{\prime}\right)\right) \\
\ell_{3}\left(t_{3}^{\prime \prime}\right)=\psi_{3}\left(\ell_{1}\left(t_{3}^{\prime \prime}\right), \ell_{2}\left(t_{3}^{\prime \prime}\right)\right)
\end{array}\right.
$$

hence

$$
\left|\ell_{3}\left(t_{3}^{\prime}\right)-\ell_{3}\left(t_{3}^{\prime \prime}\right)\right| \geq \alpha
$$

consequently

$$
\mathbf{m}\left(\ell_{3}\left(\left[t_{3}^{\prime}, t_{3}^{\prime \prime}\right]\right)\right) \geq \alpha
$$

and therefore

$$
\mathbf{m}\left(\left[t_{3}^{\prime}, t_{3}^{\prime \prime}\right]\right) \geq t_{4}
$$

On the other hand, we have: $\varphi_{3}\left(x_{1}, x_{2}\right) \leq x_{3} \leq \psi_{3}\left(x_{1}, x_{2}\right)$ and $\varphi_{3}, \psi_{3}$ are two Hölder functions on $\Omega_{3}$, i.e.,

$$
\begin{aligned}
\left|\varphi_{3}\left(x_{1}, x_{2}\right)-\varphi_{3}\left(\ell_{1}\left(t_{3}^{\prime}\right), \ell_{2}\left(t_{3}^{\prime}\right)\right)\right| & \leq h_{3}\left[\max \left\{\left|x_{1}-\ell_{1}\left(t_{3}^{\prime}\right)\right|,\left|x_{2}-\ell_{2}\left(t_{3}^{\prime}\right)\right|\right\}\right]^{\beta_{3}} \\
& \leq h_{3}\left[\max \left\{\alpha, \alpha \delta_{2}\right\}\right]^{\beta_{3}}=h_{3}\left(\alpha \delta_{2}\right)^{\beta_{3}} .
\end{aligned}
$$




$$
\begin{aligned}
\left|\psi_{3}\left(x_{1}, x_{2}\right)-\psi_{3}\left(\ell_{1}\left(t_{3}^{\prime \prime}\right), \ell_{2}\left(t_{3}^{\prime \prime}\right)\right)\right| & \leq H_{3}\left[\max \left\{\left|x_{1}-\ell_{1}\left(t_{3}^{\prime \prime}\right)\right|,\left|x_{2}-\ell_{2}\left(t_{3}^{\prime \prime}\right)\right|\right\}\right]^{\gamma_{3}} \\
& \leq H_{3}\left[\max \left\{\alpha, \alpha \delta_{2}\right\}\right]^{\gamma_{3}}=H_{3}\left(\alpha \delta_{2}\right)^{\gamma_{3}} .
\end{aligned}
$$

Hence

$$
\ell_{3}\left(t_{3}^{\prime}\right)-h_{3}\left(\alpha \delta_{2}\right)^{\beta_{3}} \leq x_{3} \leq \ell_{3}\left(t_{3}^{\prime \prime}\right)+H_{3}\left(\alpha \delta_{2}\right)^{\gamma_{3}} .
$$

Obviously, we will study the following cases:

$$
\left\{\begin{array}{l}
(4) \ell_{3}\left(t_{3}^{\prime}\right)-h_{3}\left(\alpha \delta_{2}\right)^{\beta_{3}} \leq x_{3} \leq \ell_{3}\left(t_{3}^{\prime}\right) \\
(5) \ell_{3}\left(t_{3}^{\prime}\right) \leq x_{3} \leq \ell_{3}\left(t_{3}^{\prime \prime}\right) \\
(6) \ell_{3}\left(t_{3}^{\prime \prime}\right) \leq x_{3} \leq \ell_{3}\left(t_{3}^{\prime \prime}\right)+H_{3}\left(\alpha \delta_{2}\right)^{\gamma_{3}} .
\end{array}\right.
$$

If (4) holds, we take $\mathbb{I}_{3}=\left[t_{3}^{\prime}, t_{3}^{\prime}+t_{4}\right]$.

If (5) is satisfied, there exists $\mathbb{I}_{3} \subset\left[t_{3}^{\prime}, t_{3}^{\prime \prime}\right]$ such that:

$$
\ell_{3}\left(I_{3}\right)=\left[x_{3}-\alpha, x_{3}+\alpha\right] \cap\left[\ell_{3}\left(t_{3}^{\prime}\right), \ell_{3}\left(t_{3}^{\prime \prime}\right)\right]
$$

If the condition (6) is valid, we take $\mathbb{I}_{3}=\left[t_{3}^{\prime \prime}-t_{4}, t_{3}^{\prime \prime}\right]$.

Hence, we have $\mathbb{I}_{3} \subset\left[t_{3}^{\prime}, t_{3}^{\prime \prime}\right]$ and $\mathbf{m}\left(\mathbb{I}_{3}\right) \geq t_{4}$ in all cases. In addition, for all $t \in \mathbb{I}_{3}$, we have: If (4) holds:

$$
\left|x_{3}-\ell_{3}(t)\right| \leq\left|x_{3}-\ell_{3}\left(t_{3}^{\prime}\right)\right|+\left|\ell_{3}\left(t_{3}^{\prime}\right)-\ell_{3}(t)\right| \leq \alpha+h_{3}\left(\alpha \delta_{2}\right)^{\beta_{3}}
$$

If (5) is satisfied, then:

$$
\left|x_{3}-\ell_{3}(t)\right| \leq \alpha
$$

If the condition (6) holds:

$$
\left|x_{3}-\ell_{3}(t)\right| \leq\left|x_{3}-\ell_{3}\left(t_{3}^{\prime \prime}\right)\right|+\left|\ell_{3}\left(t_{3}^{\prime \prime}\right)-\ell_{3}(t)\right| \leq \alpha+H_{3}\left(\alpha \delta_{2}\right)^{\gamma_{3}} .
$$

Then in all cases, we have for every $t \in \mathbb{I}_{3}$

$$
\left|x_{3}-\ell_{3}(t)\right| \leq \alpha\left\{1+\max \left(h_{3} \alpha^{\beta_{3}-1} \delta_{2}^{\beta_{3}}, H_{3} \alpha^{\gamma_{3}-1} \delta_{2}^{\gamma_{3}}\right)\right\}
$$

i.e.,

$$
\left|x_{3}-\ell_{3}(t)\right| \leq \alpha \delta_{3}
$$

With the same process given previously until the step $(n-1)$, we obtain by induction for all $t \in \mathbb{I}_{n-1}$,

$$
\left|x_{n-1}-\ell_{n-1}(t)\right| \leq \alpha \delta_{n-1},
$$

with $\mathbf{m}\left(\mathbb{I}_{n-1}\right) \geq t_{n}$ and $\mathbb{I}_{n-1} \subset \mathbb{I}_{n-2} \subset \cdots \subset \mathbb{I}_{1} \subset \mathbb{I}$.

Therefore, there exist $t_{n}^{\prime}, t_{n}^{\prime \prime} \in \mathbb{I}_{n-1}$ such that:

$$
\left\{\begin{array}{l}
\ell_{n}\left(t_{n}^{\prime}\right)=\varphi_{n}\left(\ell_{1}\left(t_{n}^{\prime}\right), \ldots, \ell_{n-1}\left(t_{n}^{\prime}\right)\right) \\
\ell_{n}\left(t_{n}^{\prime \prime}\right)=\psi_{n}\left(\ell_{1}\left(t_{n}^{\prime \prime}\right), \ldots, \ell_{n-1}\left(t_{n}^{\prime \prime}\right)\right) .
\end{array}\right.
$$

On the other hand, according to the definition of the feasible region, we have:

$$
\varphi_{n}\left(x_{1}, \ldots, x_{n-1}\right) \leq x_{n} \leq \psi_{n}\left(x_{1}, \ldots, x_{n-1}\right.
$$


Moreover, the two functions $\varphi_{n}, \psi_{n}$ are Hölderian on the hyper-rectangle $\Omega_{n}$, and as shown in section 2 , the sequence of numbers $\left(\delta_{i}\right)_{1 \leq i \leq n}$ is increasing, we have

$$
\begin{aligned}
& \left|\varphi_{n}\left(x_{1}, \ldots, x_{n-1}\right)-\varphi_{n}\left(\ell_{1}\left(t_{n}^{\prime}\right), \ldots, \ell_{n-1}\left(t_{n}^{\prime}\right)\right)\right| \\
& \leq h_{n}\left[\max \left\{\left|x_{1}-\ell_{1}\left(t_{n}^{\prime}\right)\right|, \ldots,\left|x_{n-1}-\ell_{n-1}\left(t_{n}^{\prime}\right)\right|\right\}\right]^{\beta_{n}} \\
& \leq h_{n}\left[\max \left\{\alpha, \alpha \delta_{2}, \ldots, \alpha \delta_{n-1}\right\}\right]^{\beta_{n}}=h_{n}\left(\alpha \delta_{n-1}\right)^{\beta_{n}} . \\
& \left|\psi_{n}\left(x_{1}, \ldots, x_{n-1}\right)-\psi_{n}\left(\ell_{1}\left(t_{n}^{\prime \prime}\right), \ldots, \ell_{n-1}\left(t_{n}^{\prime \prime}\right)\right)\right| \\
& \leq H_{n}\left[\max \left\{\alpha, \alpha \delta_{2}, \ldots, \alpha \delta_{n-1}\right\}\right]^{\gamma_{n}}=H_{n}\left(\alpha \delta_{n-1}\right)^{\gamma_{n}} .
\end{aligned}
$$

Hence

$$
\ell_{n}\left(t_{n}^{\prime}\right)-h_{n}\left(\alpha \delta_{n-1}\right)^{\beta_{n}} \leq x_{n} \leq \ell_{n}\left(t_{n}^{\prime \prime}\right)+H_{n}\left(\alpha \delta_{n-1}\right)^{\gamma_{n}} .
$$

Again, we have three cases to check:

$$
\left\{\begin{array}{l}
(7) \ell_{n}\left(t_{n}^{\prime}\right)-h_{n}\left(\alpha \delta_{n-1}\right)^{\beta_{n}} \leq x_{n} \leq \ell_{n}\left(t_{n}^{\prime}\right) \\
(8) \ell_{n}\left(t_{n}^{\prime}\right) \leq x_{n} \leq \ell_{n}\left(t_{n}^{\prime \prime}\right) \\
(9) \ell_{n}\left(t_{n}^{\prime \prime}\right) \leq x_{n} \leq \ell_{n}\left(t_{n}^{\prime \prime}\right)+H_{n}\left(\alpha \delta_{n-1}\right)^{\gamma_{n}} .
\end{array}\right.
$$

If (7) holds, we have:

$$
\begin{gathered}
\left|x_{n}-\ell_{n}\left(t_{n}^{\prime}\right)\right| \leq h_{n}\left(\alpha \delta_{n-1}\right)^{\beta_{n}}, \\
\qquad \begin{aligned}
& \text { and } \\
&\left\|\max -\ell_{\alpha}\left(t_{n}^{\prime}\right)\right\|=\max \left\{\begin{array}{l}
\left.\left|x_{1}-\ell_{1}\left(t_{n}^{\prime}\right)\right|, \ldots,\left|x_{n-1}-\ell_{n-1}\left(t_{n}^{\prime}\right)\right|,\left|x_{n}-\ell_{n}\left(t_{n}^{\prime}\right)\right|\right\} \\
\alpha, \alpha \delta_{2}, \ldots, \alpha \delta_{n-1}, h_{n}\left(\alpha \delta_{n-1}\right)^{\beta_{n}}
\end{array}\right\} \\
& \leq \max \left\{\alpha \delta_{n-1}, h_{n}\left(\alpha \delta_{n-1}\right)^{\beta_{n}}\right\} .
\end{aligned}
\end{gathered}
$$

If (8) is satisfied, there exists $t_{0} \in \mathbb{I}_{n-1}$ such that $x_{n}=\ell_{n}\left(t_{0}\right)$, consequently

$$
\begin{aligned}
\left\|x-\ell_{\alpha}\left(t_{0}\right)\right\| & =\max \left\{\left|x_{1}-\ell_{1}\left(t_{0}\right)\right|, \ldots,\left|x_{n-1}-\ell_{n-1}\left(t_{0}\right)\right|,\left|x_{n}-\ell_{n}\left(t_{0}\right)\right|\right\} \\
& \leq \max \left\{\alpha, \alpha \delta_{2}, \ldots, \alpha \delta_{n-1}, \alpha\right\} \\
& \leq \alpha \delta_{n-1} .
\end{aligned}
$$

If the last condition (9) holds, we have:

$$
\left|x_{n}-\ell_{n}\left(t_{n}^{\prime \prime}\right)\right| \leq H_{n}\left(\alpha \delta_{n-1}\right)^{\gamma_{n}},
$$

$$
\begin{aligned}
& \left\|\mid x-\ell_{\alpha}\left(t_{n}^{\prime \prime}\right)\right\|=\max \left\{\left|x_{1}-\ell_{1}\left(t_{n}^{\prime \prime}\right)\right|, \ldots,\left|x_{n-1}-\ell_{n-1}\left(t_{n}^{\prime \prime}\right)\right|,\left|x_{n}-\ell_{n}\left(t_{n}^{\prime \prime}\right)\right|\right\} \\
& \leq \max \left\{\alpha, \alpha \delta_{2}, \ldots, \alpha \delta_{n-1}, H_{n}\left(\alpha \delta_{n-1}\right)^{\gamma_{n}}\right\} \\
& \leq \max \left\{\alpha \delta_{n-1}, H_{n}\left(\alpha \delta_{n-1}\right)^{\gamma_{n}}\right\} \text {. }
\end{aligned}
$$

We deduce that the parameterized curve $\ell_{\alpha}(t)=\left(\ell_{1}(t), \ldots, \ell_{n}(t)\right)$ for $t \in \mathbb{I}$, is $\delta_{n-1} \lambda_{n} \alpha$ dense in $D$.

Corollary 1. Let $\ell_{\alpha}=\left(\ell_{1}, \ldots, \ell_{n}\right): \mathbb{I} \rightarrow D$, be a continuous function, whose components $\ell_{i},(i=1, \ldots, n)$ are Hölder functions with constants $c_{i}>0$ and exponents $\mu_{i}<1,(i=1, \ldots, n)$ (resp.). Let $t_{2}, t_{3}, \ldots, t_{n}, \alpha$ be strictly positive numbers such that:

1) $\ell_{1}$ is surjective,

2) For all $i=2, \ldots, n$ and for all interval $\mathbb{I}_{i-1}$ with length $t_{i}$, there exist $t_{i}^{\prime}$ and $t_{i}^{\prime \prime} \in \mathbb{I}_{i-1}$ such that:

$$
\left\{\begin{array}{l}
\ell_{i}\left(t_{i}^{\prime}\right)=\varphi_{i}\left(\ell_{1}\left(t_{i}^{\prime}\right), \ldots, \ell_{i-1}\left(t_{i}^{\prime}\right)\right) \\
\ell_{i}\left(t_{i}^{\prime \prime}\right)=\psi_{i}\left(\ell_{1}\left(t_{i}^{\prime \prime}\right), \ldots, \ell_{i-1}\left(t_{i}^{\prime \prime}\right)\right)
\end{array}\right.
$$


3) For all $i=2, \ldots, n$

$$
c_{i-1}<\frac{\alpha}{t_{i}^{\mu_{i-1}}}
$$

Then, the parameterized curve defined by $\ell_{\alpha}(t)=\left(\ell_{1}(t), \ldots, \ell_{n}(t)\right)$ for $t \in \mathbb{I}$, is $\delta_{n-1} \lambda_{n} \alpha-$ dense in $D$.

Proof. It is a consequence of Theorem 1. The conditions 1) and 2) of the Theorem 1 are satisfied. It remains to check the condition 3). Indeed, let $i=2, \ldots, n$ and $\mathbb{I}_{i-1}$ an interval of $\mathbb{I}$ satisfying $\mathbf{m}\left(\mathbb{I}_{i-1}\right)<t_{i}$. For $t^{\prime}, t^{\prime \prime} \in \mathbb{I}_{i-1}$, we have:

$$
\left|\ell_{i-1}\left(t^{\prime}\right)-\ell_{i-1}\left(t^{\prime \prime}\right)\right| \leq c_{i-1}\left|t^{\prime}-t^{\prime \prime}\right|^{\mu_{i-1}} \leq c_{i-1} \mathbf{m}(I)^{\mu_{i-1}}
$$

involving

$$
\mathbf{m}\left(\ell_{i-1}\left(\mathbb{I}_{i-1}\right)\right) \leq c_{i-1} \mathbf{m}\left(\mathbb{I}_{i-1}\right)^{\mu_{i-1}}<c_{i-1} t_{i}^{\mu_{i-1}}<\alpha
$$

\subsection{Constructing a concrete $\alpha$-dense curves}

In this subsection, we will use the following notations:

$$
\begin{aligned}
& \Phi_{1}(t)=a \text { and } \Phi_{i}(t)=\varphi_{i}\left(\ell_{1}(t), \ldots, \ell_{i-1}(t)\right) \text { for } i \geq 2 \\
& \Psi_{1}(t)=b \text { and } \Psi_{i}(t)=\psi_{i}\left(\ell_{1}(t), \ldots, \ell_{i-1}(t)\right) \text { for } i \geq 2,
\end{aligned}
$$

where $\varphi_{i}$ and $\psi_{i}$ for $i \geq 2$, are the functions defined in the feasible region (3).

Theorem 2. Consider the compact $D$ given in the problem (1) and let the parameterized curve $\ell_{\alpha}=\left(\ell_{1}, \ldots, \ell_{n}\right): \mathbb{I} \rightarrow D$ be defined by

$$
\ell_{i}(t)=\Phi_{i}(t) \cos ^{2}\left(\frac{\alpha_{i} t}{2}\right)+\Psi_{i}(t) \sin ^{2}\left(\frac{\alpha_{i} t}{2}\right) \text { for } i=1, \ldots, n,
$$

where $\alpha_{1}, \ldots, \alpha_{n}, \alpha$ are strictly positive numbers satisfying the relationship

$$
\left(\alpha_{i} / \pi\right)^{\beta_{1} \ldots \beta_{i-1}} \geq(1 / 2 \alpha)\left(\left(U_{i-1}-L_{i-1}\right) \alpha_{i-1}+2\left(h_{i-1} z_{i-2}^{\beta_{i-1}}+H_{i-1} z_{i-2}^{\gamma_{i-1}}\right)\right)
$$

with

$$
z_{i}=\max _{1 \leq k \leq i}\left(\frac{1}{2}\left(U_{k}-L_{k}\right) \alpha_{k}+\left(h_{k} z_{k-1}^{\beta_{k}}+H_{k} z_{k-1}^{\gamma_{k}}\right)\right), \quad z_{0}=0
$$

and $L_{i}, U_{i}$ are respectively the lower and upper bounds of the functions $\varphi_{i}$ and $\psi_{i}$ on the hyper-rectangles $\Omega_{i}$ (see section 2). Then, the parameterized curve $\ell_{\alpha}(t)=\left(\ell_{1}(t), \ldots, \ell_{n}(t)\right)$ for $t \in\left[0, \pi / \alpha_{1}\right]$ is $\delta_{n-1} \lambda_{n} \alpha$-dense in $D$.

Proof. This result is a consequence of Theorem 1. Indeed, the proof is obtained in a recurrent way:

(i) For $i=2$.

1) $\ell_{1}$ is surjective by definition.

2) If we take $t_{2}=\frac{\pi}{\alpha_{2}}$, then for all interval $\mathbb{I}_{1}$ with length $t_{2}$, there exist $t^{\prime}, t^{\prime \prime} \in \mathbb{I}_{1}$ such that:

$$
\begin{aligned}
&\left\{\begin{array} { l } 
{ \ell _ { 2 } ( t ^ { \prime } ) = \varphi _ { 2 } ( \ell _ { 1 } ( t ^ { \prime } ) ) } \\
{ \ell _ { 2 } ( t ^ { \prime \prime } ) = \psi _ { 2 } ( \ell _ { 1 } ( t ^ { \prime \prime } ) ) }
\end{array} \Rightarrow \left\{\begin{array}{l}
\cos ^{2}\left(\alpha_{2} t^{\prime} / 2\right)=1 \\
\sin ^{2}\left(\alpha_{2} t^{\prime \prime} / 2\right)=1
\end{array}\right.\right. \\
& \Rightarrow\left\{\begin{array}{l}
t^{\prime}=2 \pi k / \alpha_{2} \\
t^{\prime \prime}=(2 k+1) \pi / \alpha_{2}
\end{array}, k \in \mathbb{N} .\right.
\end{aligned}
$$


3) For any interval $\mathbb{I}_{1}$ satisfying $\mathbf{m}\left(\mathbb{I}_{1}\right)<\frac{\pi}{\alpha_{2}}$, we have $\mathbf{m}\left(\ell_{1}\left(\mathbb{I}_{1}\right)\right)<\alpha$. $\forall t^{\prime}, t^{\prime \prime} \in \mathbb{I}_{1}:$

$$
\begin{aligned}
\left|\ell_{1}\left(t^{\prime}\right)-\ell_{1}\left(t^{\prime \prime}\right)\right| & =\left|a\left(\cos ^{2}\left(\alpha_{1} t^{\prime} / 2\right)-\cos ^{2}\left(\alpha_{1} t^{\prime \prime} / 2\right)\right)+b\left(\sin ^{2}\left(\alpha_{1} t^{\prime} / 2\right)-\sin ^{2}\left(\alpha_{1} t^{\prime \prime} / 2\right)\right)\right| \\
& \leq(b-a)\left|\sin \frac{\alpha_{1} t^{\prime}-\alpha_{1} t^{\prime \prime}}{2}\right| \leq \frac{1}{2}(b-a) \alpha_{1}\left|t^{\prime}-t^{\prime \prime}\right| \leq \frac{1}{2}(b-a) \alpha_{1} \frac{\pi}{\alpha_{2}} \leq \alpha .
\end{aligned}
$$

By Theorem 1, the parameterized curve $\ell(t)=\left(\ell_{1}(t), \ell_{2}(t)\right)$ defined on $\left[0, \pi / \alpha_{1}\right]$ is $\lambda_{2} \alpha$ dense in $D$ with $\frac{\alpha_{2}}{\pi} \geq \frac{(b-a) \alpha_{1}}{2 \alpha}$.

(ii) We suppose that the theorem is true for $i$.

2) For all $i=2, \ldots, n$ and for all interval $\mathbb{I}_{i-1}$ with length $t_{i}=\frac{\pi}{\alpha_{i}}$, there exist $t_{i}^{\prime}=\frac{2 \pi k}{\alpha_{i}}$ and $t_{i}^{\prime \prime}=\frac{\pi}{\alpha_{i}}+\frac{2 \pi k}{\alpha_{i}} \in \mathbb{I}_{i-1} ; k \in \mathbb{N}$, such that:

$$
\left\{\begin{array}{l}
\ell_{i}\left(t^{\prime}\right)=\varphi_{i}\left(\ell_{1}\left(t^{\prime}\right), \ldots, \ell_{i-1}\left(t^{\prime}\right)\right) \\
\ell_{i}\left(t^{\prime \prime}\right)=\psi_{i}\left(\ell_{1}\left(t^{\prime \prime}\right), \ldots, \ell_{i-1}\left(t^{\prime \prime}\right)\right)
\end{array}\right.
$$

3) We suppose that the inequality holds for $i=2, \ldots, n$, i.e., for any closed interval $\mathbb{J}$ of length less than $\frac{\pi}{\alpha_{i}}$; we have:

$$
\mathbf{m}(\mathbb{J})<\frac{\pi}{\alpha_{i}} \Rightarrow \mathbf{m}\left(\ell_{i-1}(\mathbb{J})\right)<\alpha .
$$

Consequently

$$
\begin{aligned}
& \forall t^{\prime}, t^{\prime \prime} \in \mathbb{J}:\left|\ell_{i-1}\left(t^{\prime}\right)-\ell_{i-1}\left(t^{\prime \prime}\right)\right| \leq \\
& {\left[\frac{1}{2}\left(U_{i-1}-L_{i-1}\right) \alpha_{i-1}+\left(h_{i-1} z_{i-1}^{\beta_{i-1}}+H_{i-1} z_{i-1}^{\gamma_{i-1}}\right)\right]\left|t^{\prime}-t^{\prime \prime}\right|^{\beta_{1} \ldots \beta_{i-1}} \leq \alpha .}
\end{aligned}
$$

Consider the closed interval $\mathbb{J}$, such that: $\mathbf{m}(\mathbb{J})<\frac{\pi}{\alpha_{i+1}}$, show that for $t^{\prime}, t^{\prime \prime} \in \mathbb{J}$, we have:

$$
\begin{aligned}
& \left|\ell_{i}\left(t^{\prime}\right)-\ell_{i}\left(t^{\prime \prime}\right)\right|=\mid \begin{array}{c}
\Phi_{i}\left(t^{\prime}\right) \cos ^{2}\left(\alpha_{i} t^{\prime} / 2\right)+\Psi_{i}\left(t^{\prime}\right) \sin ^{2}\left(\alpha_{i} t^{\prime} / 2\right)- \\
\Phi_{i}\left(t^{\prime \prime}\right) \cos ^{2}\left(\alpha_{i} t^{\prime \prime} / 2\right)-\Psi_{i}\left(t^{\prime \prime}\right) \sin ^{2}\left(\alpha_{i} t^{\prime \prime} / 2\right)
\end{array} \\
& +\left(\Phi_{i}\left(t^{\prime}\right)-\Psi_{i}\left(t^{\prime}\right)\right) \cos ^{2}\left(\alpha_{i} t^{\prime \prime} / 2\right)-\left(\Phi_{i}\left(t^{\prime}\right)-\Psi_{i}\left(t^{\prime}\right)\right) \cos ^{2}\left(\alpha_{i} t^{\prime \prime} / 2\right) \mid \\
& =\mid\left(\Phi_{i}\left(t^{\prime}\right)-\Psi_{i}\left(t^{\prime}\right)\right)\left(\cos ^{2}\left(\alpha_{i} t^{\prime} / 2\right)-\cos ^{2}\left(\alpha_{i} t^{\prime \prime} / 2\right)\right)+\left(\Phi_{i}\left(t^{\prime}\right)-\Phi_{i}\left(t^{\prime \prime}\right)\right)\left(\cos ^{2}\left(\alpha_{i} t^{\prime \prime} / 2\right)\right. \\
& \left(\Psi_{i}\left(t^{\prime}\right)-\Psi_{i}\left(t^{\prime \prime}\right) \sin ^{2}\left(\alpha_{i} t^{\prime \prime} / 2\right) \mid\right. \\
& \leq\left|\Phi_{i}\left(t^{\prime}\right)-\Psi_{i}\left(t^{\prime}\right)\right|\left|\cos ^{2}\left(\alpha_{i} t^{\prime} / 2\right)-\cos ^{2}\left(\alpha_{i} t^{\prime \prime} / 2\right)\right|+\left|\Phi_{i}\left(t^{\prime}\right)-\Phi_{i}\left(t^{\prime \prime}\right)\right|+\left|\Psi_{i}\left(t^{\prime}\right)-\Psi_{i}\left(t^{\prime \prime}\right)\right| .
\end{aligned}
$$

Let us take into account that the constants $L_{i}, U_{i}$ are respectively the lower and upper bounds of the functions $\varphi_{i}, \psi_{i}$ on the hyper-rectangles $\Omega_{i}$ (as shown in section 2). If we suppose that $\gamma_{i} \geq \beta_{i}$ (or $\gamma_{i}<\beta_{i}$ ) for each $i$, then we have

$$
\begin{aligned}
& \left|\ell_{i}\left(t^{\prime}\right)-\ell_{i}\left(t^{\prime \prime}\right)\right| \leq \frac{1}{2}\left(U_{i}-L_{i}\right) \alpha_{i}\left|t^{\prime}-t^{\prime \prime}\right|+h_{i}\left(\max \left\{\left|\ell_{1}\left(t^{\prime}\right)-\ell_{1}\left(t^{\prime \prime}\right)\right|, \ldots,\left|\ell_{i-1}\left(t^{\prime}\right)-\ell_{i-1}\left(t^{\prime \prime}\right)\right|\right\}\right)^{\beta_{i}}+ \\
& +H_{i}\left(\max \left\{\left|\ell_{1}\left(t^{\prime}\right)-\ell_{1}\left(t^{\prime \prime}\right)\right|, \ldots,\left|\ell_{i-1}\left(t^{\prime}\right)-\ell_{i-1}\left(t^{\prime \prime}\right)\right|\right\}\right)^{\gamma_{i}} \\
& \leq \frac{1}{2}\left(U_{i}-L_{i}\right) \alpha_{i}\left|t^{\prime}-t^{\prime \prime}\right|+ \\
& h_{i}\left(\max \left\{\frac{1}{2}(b-a) \alpha_{1}, \ldots, \frac{1}{2}\left(U_{i-1}-L_{i-1}\right) \alpha_{i-1}+\left(h_{i-1} z_{i-2}^{\beta_{i-1}}+H_{i-1} z_{i-2}^{\gamma_{i-1}}\right)\right\}\left|t^{\prime}-t^{\prime \prime}\right|^{\beta_{1} \ldots \beta_{i-1}}\right)^{\beta_{i}}
\end{aligned}
$$




$$
\begin{aligned}
& +H_{i}\left(\max \left\{\frac{1}{2}(b-a) \alpha_{1}, \ldots, \frac{1}{2}\left(U_{i-1}-L_{i-1}\right) \alpha_{i-1}+\left(h_{i-1} z_{i-2}^{\beta_{i-1}}+H_{i-1} z_{i-2}^{\gamma_{i-1}}\right)\right\}\left|t^{\prime}-t^{\prime \prime}\right|^{\beta_{1} \ldots \beta_{i-1}}\right)^{\gamma_{i}} \\
& \leq\left[\frac{1}{2}\left(U_{i}-L_{i}\right) \alpha_{i}+\left(h_{i} z_{i-1}^{\beta_{i}}+H_{i} z_{i-1}^{\gamma_{i}}\right)\right]\left|t^{\prime}-t^{\prime \prime}\right|^{\beta_{1} \ldots \beta_{i}} \\
& <\left[\frac{1}{2}\left(U_{i}-L_{i}\right) \alpha_{i}+\left(h_{i} z_{i-1}^{\beta_{i}}+H_{i} z_{i-1}^{\gamma_{i}}\right)\right]\left(\frac{\pi}{\alpha_{i+1}}\right)^{\beta_{1} \ldots \beta_{i}}<\alpha
\end{aligned}
$$

Hence the inequality holds for $i+1$.

Remark 2. All the functions $\ell_{i}(t)$ defined in Theorem 2 are Hölderian with constants $c_{i}>0$ and exponents $\mu_{i}<1$, for each $i=1, \ldots, n$, given by

$$
\left\{\begin{array}{l}
c_{i}=\frac{1}{2}\left(U_{i}-L_{i}\right) \alpha_{i}+\left(h_{i} z_{i-1}^{\beta_{i}}+H_{i} z_{i-1}^{\gamma_{i}}\right) \\
\mu_{i}=\beta_{1} \ldots \beta_{i}
\end{array}\right.
$$

It is well known that if a function with several variables $F(x)$ is Hölderian of the constant $H$ and the exponent $\beta^{\prime}$ on the compact $\Omega$, then the function of a single variable $f(t)=F\left(\ell_{\alpha}(t)\right)$ is Hölderian with the constant $h=H\left(\max _{1 \leq i \leq n} c_{i}\right)^{\beta^{\prime}}$ and exponent $\beta=\beta^{\prime}\left(\min _{1 \leq i \leq n} \mu_{i}\right)$ on the interval $\left[0, \pi / \alpha_{1}\right]$.

\subsubsection{Examples of a compact sets which are $\alpha$-densifiable}

In this subsection and according to Theorem 2, the illustrations Figures 1-5 given below, represent a densification of some non-convex sets and hyper-rectangles (for $n=2$ and $n=3$ ), by the supports of the different $\alpha$-dense curves $\ell_{\alpha}(t)$ on $[0, \pi]$ (with $\alpha_{1}=1$ ). Note that the parameterized curves obtained in the examples $D_{1}, D_{3}$ and $D_{4}$ are regular of class $\mathcal{C}^{\infty}$ whereas the curve in $D_{2}$, is only continuous.

a) Examples with non-convex sets

Let $D_{1}=\left\{\left(x_{1}, x_{2}\right) \in \mathbb{R}^{2} /-1 \leq x_{1} \leq 1,-\frac{1}{4} x_{1}^{2}+\frac{1}{2} x_{1}-1 \leq x_{2} \leq \frac{1}{4} x_{1}^{2}+\frac{1}{2} x_{1}+1\right\}$, here, we have $\beta_{2}=\gamma_{2}=1$.

$$
\begin{aligned}
& D_{2}=\left\{\left(x_{1}, x_{2}\right) \in \mathbb{R}^{2} / \quad x_{1}^{2}+x_{2}^{2} \leq 1, \quad\left|x_{1}\right|-\left(x_{2}+1\right)^{2} \leq 0\right\}, \\
& \varphi_{2}\left(x_{1}\right)=\sqrt{\left|x_{1}\right|}-1 \text { and } \psi_{2}\left(x_{1}\right)=\sqrt{1-x_{1}^{2}} \text { and } \beta_{2}=\gamma_{2}=1 / 2 .
\end{aligned}
$$

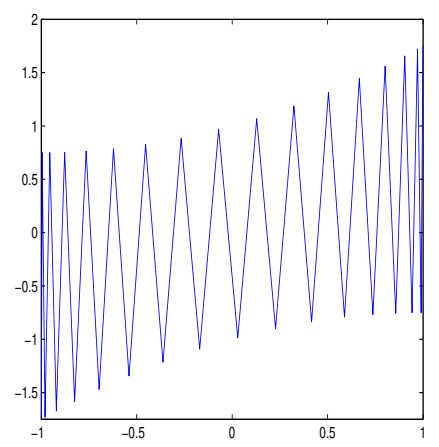

Figure 1: The $\alpha$-dense curve $\ell_{\alpha}$ in $D_{1}$ with $\alpha=0.2$ 


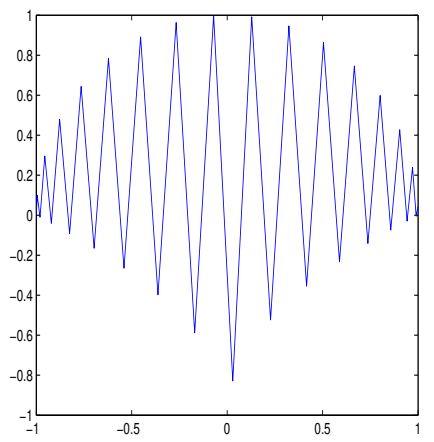

Figure 2: The $\alpha$-dense curve $\ell_{\alpha}$ in $D_{2}$ with $\alpha=0.2$

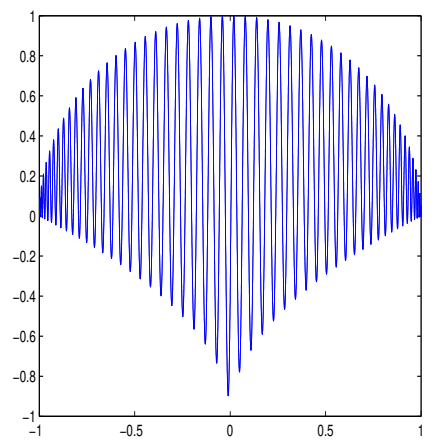

Figure 3: The $\alpha$-dense curve $\ell_{\alpha}$ in $D_{2}$ with $\alpha=0.01$

Remark 3. In particular, if the functions $\varphi_{i}$ and $\psi_{i}(i \geq 2)$ in the definition of the feasible set $D$, are all constants and if $D=\Omega$, i.e.,

$$
\begin{aligned}
& a=x_{1}^{l} \text { and } \varphi_{i}\left(x_{1}, \ldots, x_{i-1}\right)=x_{i}^{l}, 2 \leq i \leq n, \\
& b=x_{1}^{u} \text { and } \psi_{i}\left(x_{1}, \ldots, x_{i-1}\right)=x_{i}^{u}, 2 \leq i \leq n .
\end{aligned}
$$

Then the feasible set $D$ becomes a hyper-rectangle of $\mathbb{R}^{n}$. According to Theorem 2 , the hyperrectangle $D$ is $\alpha$-densified by the parameterized curve $\ell_{\alpha}=\left(\ell_{1}, \ldots, \ell_{n}\right)$ defined on $\left[0, \pi / \alpha_{1}\right]$, by the following expression:

$$
\ell_{i}(t)=\left(\frac{x_{i}^{l}-x_{i}^{u}}{2}\right) \cos ^{2}\left(\frac{\alpha_{i} t}{2}\right)+x_{i}^{u}, 1 \leq i \leq n,
$$

where the constants $\alpha_{1}, \ldots, \alpha_{n}, \alpha$ satisfying the relationship:

$$
\left(\frac{\alpha_{i}}{\pi}\right) \geq\left(\frac{\alpha_{i-1}}{2 \alpha}\right)\left(x_{i-1}^{u}-x_{i-1}^{l}\right), 2 \leq i \leq n .
$$

But in the implementation of the dimensionality reduction method, one can choose these last constants as:

$$
\alpha_{1}=1, \alpha_{i}=\left(\frac{\pi}{\alpha}\right) \prod_{k=1}^{i-1} \prod_{k}^{i-1}\left(x_{k}^{u}-x_{k}^{l}\right), 2 \leq i \leq n .
$$


b) Examples with the hyper-rectangles

Let $D_{3}=\left\{\left(x_{1}, x_{2}\right) \in \mathbb{R}^{2} /-1 \leq x_{1} \leq 1,-1 \leq x_{2} \leq 1\right\}$, here, we have $\varphi_{2}\left(x_{1}\right)=-1$ and $\psi_{2}\left(x_{1}\right)=1$.

$D_{4}=\left\{\left(x_{1}, x_{2}, x_{3}\right) \in \mathbb{R}^{3} /-1 \leq x_{i} \leq 1, i=1,2,3\right\}$, here, we have $\varphi_{2}\left(x_{1}\right)=-1, \psi_{2}\left(x_{1}\right)=$ $1, \varphi_{3}\left(x_{1}, x_{2}\right)=-1, \psi_{3}\left(x_{1}, x_{2}\right)=1$.

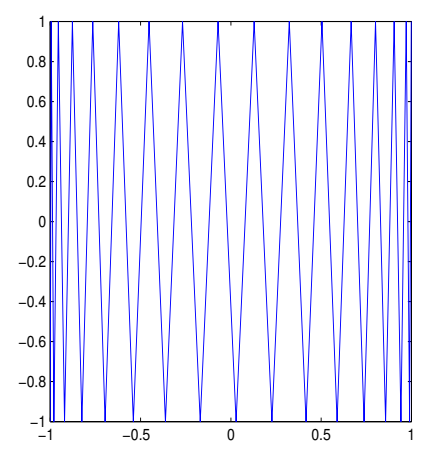

Figure 4: The $\alpha$-dense curve $\ell_{\alpha}$ in the square $D_{3}$ with $\alpha=0.2$

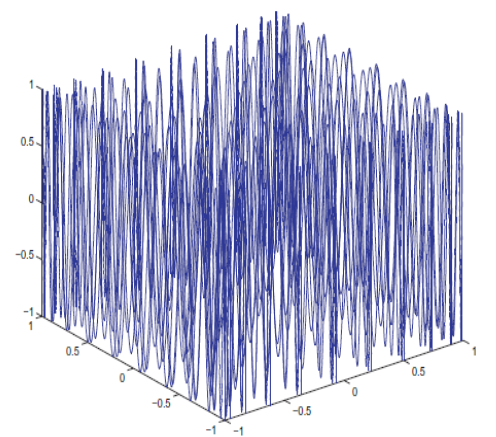

Figure 5: The $\alpha$-dense curve $\ell_{\alpha}$ in the cube $D_{4}$ with $\alpha=0.3$

Remark 4. At the end of this section, it should be mentioned that, in the dimensionality reduction method (DR), the number of evaluation points necessary for reaching the global minimum, with the accuracy $\varepsilon$, of the univariate objective function $f(t)=F\left(\ell_{\alpha}(t)\right)$, depends on the length $\mathcal{L}_{\alpha, \ell}$ of the $\alpha$-dense curve $\ell_{\alpha}$ in the feasible set, which allows to convert the constrained multi-dimensional problem (1). to the one-dimensional unconstrained one. As for all $\alpha>0$, the set of $\alpha$-dense curves in a compact $D$ of $\mathbb{R}^{n}$, contains a curve with minimal length, see Ziadi et al. [25], it is therefore, very interest to find other curves with small length in order to improve the (DR) method.

\section{One-dimensional Hölder unconstrained problem}

The univariate global minimization problem of Hölder functions over a closed and bounded interval of $\mathbb{R}$ appear frequently in several life applications, for instance, the simple plant location problem under a uniform delivered price policy, see Hanjoul et al. [6] and infinite horizon optimization problems, see Kiatsupaibul et al. [9]. In [9], the authors gave a transformation 
that reduces the problem of the infinite horizon into an equivalent one-dimensional optimization problem of Hölder function over an interval of $\mathbb{R}$. Let us consider $\ell_{\alpha}(t)=\left(\ell_{1}(t), \ldots, \ell_{n}(t)\right)$ the $\delta_{n-1} \lambda_{n} \alpha$-dense curve in $D$, for $t \in\left[0, \pi / \alpha_{1}\right]$ which is obtained from Theorem 2 . Then by using the transformation $x_{i}=\ell_{i}(t)$, for $i=1, \ldots, n$, the initial problem (1) is approached by the one-dimensional unconstrained one,

$$
\min _{t \in\left[0, \pi / \alpha_{1}\right]} f(t),
$$

where $f(t)=F\left(\ell_{\alpha}(t)\right)$ is a Hölder non-differentiable function on the interval $\left[0, \pi / \alpha_{1}\right]$ with constants $h$ and the exponent $\beta<1$. In the rest of this section, we focus on the one-dimensional global minimization problem (5).

\subsection{Constructing piecewise affine under-estimator functions}

Since the objective function $f(t)$ of problem (5) is Hölderian on $\left[0, \pi / \alpha_{1}\right]$, then we have

$$
\left|f(t)-f\left(t^{\prime}\right)\right| \leq h\left|t-t^{\prime}\right|^{\beta}, \forall t, t^{\prime} \in\left[0, \pi / \alpha_{1}\right] .
$$

The one-dimensional deterministic method that we will use for solving this problem, is that of Piyavskii [16], which is the best known and more discussed in the literature among the covering global optimization methods. It applies to Lipschitz functions on a closed and bounded interval of $\mathbb{R}$ whose Lipschitz constant is known. Recall that an under-estimator of a function $f:[a, b] \rightarrow \mathbb{R}$, is a function $U:[a, b] \rightarrow \mathbb{R}$, such that for all $x \in[a, b], U(x) \leq f(x)$. In the case of Hölder functions, it follows from the Hölder condition (6) that, for all $t, t^{\prime} \in\left[0, \pi / \alpha_{1}\right]$ we have

$$
f\left(t^{\prime}\right)-h\left|t-t^{\prime}\right|^{\beta} \leq f(t)
$$

If a point $t^{\prime} \in\left[0, \pi / \alpha_{1}\right]$ is fixed, then the function

$$
U(t)=f\left(t^{\prime}\right)-h\left|t-t^{\prime}\right|^{\beta},
$$

is an under-estimator function for $f$ on $\left[0, \pi / \alpha_{1}\right]$, i.e.,

$$
U(t) \leq f(t), \text { for all } t \in\left[0, \pi / \alpha_{1}\right]
$$

A sequence of points, $t_{1}, t_{2}, \ldots, t_{k}$, converging to the global minimizer of $f$ on $\left[0, \pi / \alpha_{1}\right]$, is generated by constructing a sequence of an under-estimator functions of $f$ over $\left[0, \pi / \alpha_{1}\right]$. In the starting step, a sampling point $t_{1}$ is chosen at the mid-point of the interval $\left[0, \pi / \alpha_{1}\right]$ as $t_{1}=\pi / 2 \alpha_{1}$, and at $t_{1}$ we have the under-estimator $U_{1}(t)=f\left(t_{1}\right)-h\left|t-t_{1}\right|^{\beta}$, which represented by two parabolic curves as shown in Figure 6. Let's set $t_{2}=\arg \min U_{1}\left(\left[0, \pi / \alpha_{1}\right]\right)$, we have obtained a new under-estimator of $f$, which is a piecewise parabolic function and is closer to $f$, as

$$
U_{2}(t)=\max _{i=1,2}\left\{f\left(t_{i}\right)-h\left|t-t_{i}\right|^{\beta}\right\} .
$$

At the iteration $k$, the function given by

$$
U_{k}(t)=\max _{i=1, \ldots, k}\left\{f\left(t_{i}\right)-h\left|t-t_{i}\right|^{\beta}\right\},
$$

which satisfies

$$
U_{k}(t) \leq U_{k+1}(t) \leq f(t), t \in\left[0, \pi / \alpha_{1}\right],
$$


is also an under-estimator of $f$ on $\left[0, \pi / \alpha_{1}\right]$. In fact, in this algorithm, we construct an increasing sequence of a piecewise parabolic functions which are the under-estimators of the objective function $f$. The restriction of the under-estimator $U_{k}$ on each sub-interval $\left[t_{i-1}, t_{i}\right], i=2, \ldots, k$ of $\left[0, \pi / \alpha_{1}\right]$, can be given as

$$
U_{i}(t)=\max _{i}\left\{q_{i}(t), r_{i}(t)\right\}
$$

where

$$
q_{i}(t)=f\left(t_{i-1}\right)-h\left(t-t_{i-1}\right)^{\beta} \text { and } r_{i}(t)=f\left(t_{i}\right)-h\left(t_{i}-t\right)^{\beta} .
$$

The stopping criterion for this algorithm is when one has $f\left(t_{o p t}\right)-U\left(t_{o p t}\right) \leq \varepsilon$, where $t_{\text {opt }}$ is the optimal global minimizer of a piecewise under-estimator function $U$ of the objective function $f$ on $\left[0, \pi / \alpha_{1}\right]$ and $\varepsilon$ is the required accuracy. On each sub-interval $\left[t_{i-1}, t_{i}\right], i=2, \ldots, k$ of $\left[0, \pi / \alpha_{1}\right]$, the function $U_{i}(t)$ is strictly convex and non-differentiable and its global minimum can be calculated by determining the intersection point of the two parabolic curves $q_{i}$ and $r_{i}$. Then at each iteration we must solve a non-linear algebraic equation:

$$
q_{i}(t)-r_{i}(t)=0 \text {. }
$$

Usually, it is not easy to find the explicit solution of this last equation, particularly in the case where the parameter $\beta$ is sufficiently small or where it is not as $\beta=\frac{1}{m}, m \in \mathbb{N}^{*}, m \neq 1$. We can even say that the equation (7) presents the same difficulty as a local optimization problem. The technique used by Gourdin et al. [4] is based on resolution at each step of the algorithm, the non-linear equation (7) in the case when $\beta=\frac{1}{m}$ and $m$ is the integers $2,3,4$ and $h$ is given, because it is possible to obtain the analytical expression of the intersection point of $q_{i}(t)$ and $r_{i}(t)$. Lera and Sergeyev [10] have replaced this intersection point by an intersection point of two linked segments of the parabolas $q_{i}(t)$ and $r_{i}(t)$. They even consider this technique when the constant $h$ is not given a priori. The previous techniques become very difficult to realise in the case of multivariate Hölderian functions, since the under-estimators of the objective function are also multivariate and the determination of their local minima at each iteration is the intersection of several parabolic hyper-surfaces on a hyper-rectangles of $\mathbb{R}^{n}$. In this case, the equation (7) is still non-linear and with several variables. To overcome this situation, a deterministic approach is proposed. It is composed of two parts, the first one, is to approximate both the multivariate Hölderian function $F$ of the initial problem (1) to a univariate one and the feasible domain $D$, by using a new computable parameterized $\alpha$-dense curves $\ell_{\alpha}(t)$ in $D$ as previously described. The second one, is to apply the modification of the Piyavskii's algorithm to the one-dimensional unconstrained problem (6). The technique used for the Hölder univariate minimization does not need to solve at iteration the equation $(7)$. It consists on constructing a sequence of piecewise affine under-estimators of $f$ on $\left[0, \pi / \alpha_{1}\right]$ similar to the previous procedure, which is essentially based on the following result.

Theorem 3 A real function $F$ defined on a compact set $D$ of $\mathbb{R}^{n}$ is continuous if and only if for any $\varepsilon>0$ there exists a constant $L>0$ such that for all $x, y \in D$,

$$
|F(x)-F(y)| \leq L\|x-y\|+\varepsilon .
$$

A version of the proof of this result is found in [22], but in this last one, the author assumes that the set $D$ is a convex of $\mathbb{R}^{n}$, whereas this condition is not necessary if $D$ is a compact. We have gave a simpler proof of this result in [17]. It is based on the fact that if $D$ is a compact of $\mathbb{R}^{n}$, then the set of Lipschitz functions on $D$ defined by

$$
\mathcal{L}(F, D)=\{F: D \rightarrow \mathbb{R}, \exists L>0,|F(x)-F(y)| \leq L\|x-y\|, \forall x, y \in D\},
$$

is dense in the space $\mathcal{C}(F, D)$ of continuous functions on $D$, on which the sup-norm $\|F-G\|=\sup _{x \in D}|F(x)-G(x)|$, is defined. The above result has a very important theoretical 
advantage, but in the numerical implementation of the majority of covering global optimization methods, we need to know the value of the constant $L$ in (8), unfortunately, it is usually difficult to calculate it. The algorithm of Piyavskii used in this paper is applied to a class of Hölderian functions. Since all the functions of this class defined on a compact $D$ of $\mathbb{R}^{n}$, with the knowledge of the constants $h$ and $\beta$, are continuous, then the condition (8) of the previous theorem is satisfied. However, in most cases, it is not easy to evaluate explicitly these constants. We can then, proceed to the numerical evaluation. Since theorem 3 only ensures the existence of the constant $L$, without giving us how to evaluate it. Due to this reason, several procedures can be envisaged, with the aim of applying the previous algorithm and improving the convergence. From the inequality (8), one can directly estimate the constant $L$, instead of estimating $h$ and $\beta$. The estimates of the constant $L$ have an influence on the convergence to the global minimum. Among the procedures allowing for estimating $L$, there is one, such that the constant $L$ is locally estimated in different sub-intervals of the search domain, the other procedure, is to estimate globally the constant $L$, i.e., valid for the entire search domain, until the optimal solution is obtained. More details are discussed in [17, 19, 20, 21]. Another idea that can be applied for some covering global optimization methods which use the constant $L$, is to construct an appropriate increasing sequence of a real positive constants $\left(L_{k}\right), k \in \mathbb{N}$ which satisfies the following conditions: $L_{k} \rightarrow \infty$ for $k \rightarrow \infty$ and for a given accuracy $\varepsilon>0$, there exists $j \in \mathbb{N}$, such that $L \leq L_{j}$ [27]. Consequently, the inequality (8) is holds with the constant $L_{j}$ for which the convergence to the global minimum is guaranteed [17]. As mentioned earlier, after using the $\alpha$-dense curve $\ell_{\alpha}(t)$ in the search region $D$, the univariate function $f(t)=F\left(\ell_{\alpha}(t)\right)$ is Hölderian on the interval $\left[0, \pi / \alpha_{1}\right]$, however, $f$ is certainly continuous and hence condition (8) is satisfied. In this case, there is no need to estimate the constant $L$, because here, the value of the constant $L$ is given explicitly with respect to $h, \beta$ and the accuracy $\varepsilon$. Let us give the following result and for more details of the proof, one can see [17].

Theorem 4 Let $f(t)=F\left(\ell_{\alpha}(t)\right)$ be a real univariate Hölder function with constant $h$ and exponent $\beta<1$ defined on the interval $\left[0, \pi / \alpha_{1}\right]$, then for all $\varepsilon>0$, we have:

$$
\left|f(t)-f\left(t^{\prime}\right)\right| \leq L_{\varepsilon}\left|t-t^{\prime}\right|+\varepsilon, \forall t, t^{\prime} \in\left[0, \pi / \alpha_{1}\right] .
$$

with

$$
L_{\varepsilon}=\left\{\begin{array}{cc}
\frac{\beta h^{1 / \beta}}{\varepsilon^{(1 / \beta)-1}} & \text { for } \beta \in\left\{\frac{1}{m}, m \in \mathbb{N}^{*}, m \neq 1\right\} \\
\frac{\beta h^{(1 / \beta)+1}}{\varepsilon^{1 / \beta}} & \text { for } \beta \in] 0,1\left[\backslash\left\{\frac{1}{m}, m \in \mathbb{N}^{*}\right\} .\right.
\end{array}\right.
$$

For a given $\varepsilon>0$ and for a known constants $h$ and $\beta<1$, the set of all such constant $L_{\varepsilon}$ is closed in $\mathbb{R}$. Then the smallest value theoretically always exists. In [17] we have suggested another technique for calculating a different constant than $L_{\varepsilon}$ that satisfies (9). Now, from the inequality (9), we construct an increasing sequence of piecewise affine under-estimators of the objective function $f(t)=F\left(\ell_{\alpha}(t)\right)$ on the interval $\left[0, \pi / \alpha_{1}\right]$, instead parabolic:

$$
L_{k}(t)=\max _{i=1, \ldots, k}\left\{f\left(t_{i}\right)-L_{\varepsilon}\left|t-t_{i}\right|-\varepsilon\right\},
$$

and on each sub-interval $\left[t_{i-1}, t_{i}\right], i=2, \ldots, k$ of $\left[0, \pi / \alpha_{1}\right]$, the restriction of $L_{k}$ can be given as

$$
L_{i}(t)=\max _{i}\left\{v_{i}(t), w_{i}(t)\right\}
$$

such that $v_{i}$ and $w_{i}$ are two linear functions which are drawn with slope $L_{\varepsilon}$, as shown in Figure 6 , where their expressions are given by

$$
\begin{aligned}
v_{i}(t) & =f\left(t_{i-1}\right)-L_{\varepsilon}\left(t-t_{i-1}\right)-\varepsilon, \quad t \in\left[t_{i-1}, \widetilde{t_{i}}\right] \\
w_{i}(t) & =f\left(t_{i}\right)-L_{\varepsilon}\left(t_{i}-t\right)-\varepsilon, t \in\left[\widetilde{t_{i}}, t_{i}\right] .
\end{aligned}
$$




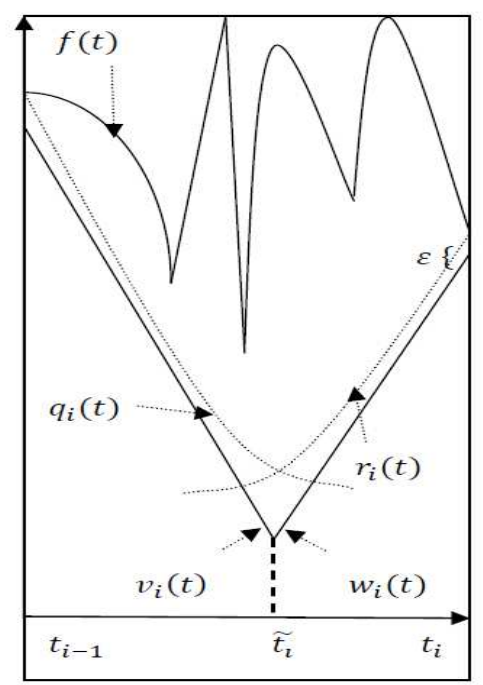

Figure 6: The piecewise affine under-estimator of $f(t)=F\left(\ell_{\alpha}(t)\right)$ on $\left[t_{i-1}, t_{i}\right]$

Note that the global minimum of the functions $L_{i}$ on the interval $\left[t_{i-1}, t_{i}\right]$, is calculated at $\widetilde{t_{i}}$ the intersection point of two lines $v_{i}$ and $w_{i}$ which is easily found by solving over the interval $\left[t_{i-1}, t_{i}\right]$, a simple linear equation with the single variable $t$ even $\beta$ is not an integer or is large:

$$
v_{i}(t)-w_{i}(t)=0
$$

The explicit solution $\widetilde{t_{i}}$ of the last equation, and the value $L_{i}\left(\widetilde{t_{i}}\right)$ are given by

$$
\left\{\begin{array}{l}
\widetilde{t_{i}}=\frac{1}{2}\left(\frac{1}{L_{\varepsilon}}\left(y_{i-1}-y_{i}\right)+t_{i-1}+t_{i}\right), i=2, \ldots, k \\
L_{i}\left(\widetilde{t_{i}}\right)=\frac{1}{2}\left(y_{i-1}+y_{i}-L_{\varepsilon}\left(t_{i}-t_{i-1}\right)-2 \varepsilon\right),
\end{array}\right.
$$

where $y_{j}=F\left(\ell_{\alpha}\left(t_{j}\right)\right), j=1, \ldots, k$. Then we have

$$
L_{i}\left(\widetilde{t_{i}}\right)=\min _{t \in\left[t_{i-1}, t_{i}\right]} L_{i}(t) \leq f(t), \forall t \in\left[t_{i-1}, t_{i}\right]
$$

The modified algorithm of Piyavskii MPA proceeds then to evaluate the global minimum of the affine under-estimator of $f(t)=F\left(\ell_{\alpha}(t)\right)$ on the search domain $\left[0, \pi / \alpha_{1}\right]$ through the construction of an increasing sequence of piecewise affine under-estimators. This algorithm can be extended to the case when the constant $L_{\varepsilon}$ is a priori unknown. Let's now show the combined method of dimensionality reduction and the modified Piyavskii's method, which is called DR-MPA, as follows:

Algorithm DR-MPA The dimensionality reduction combined with

the modified Piyavskii's method.

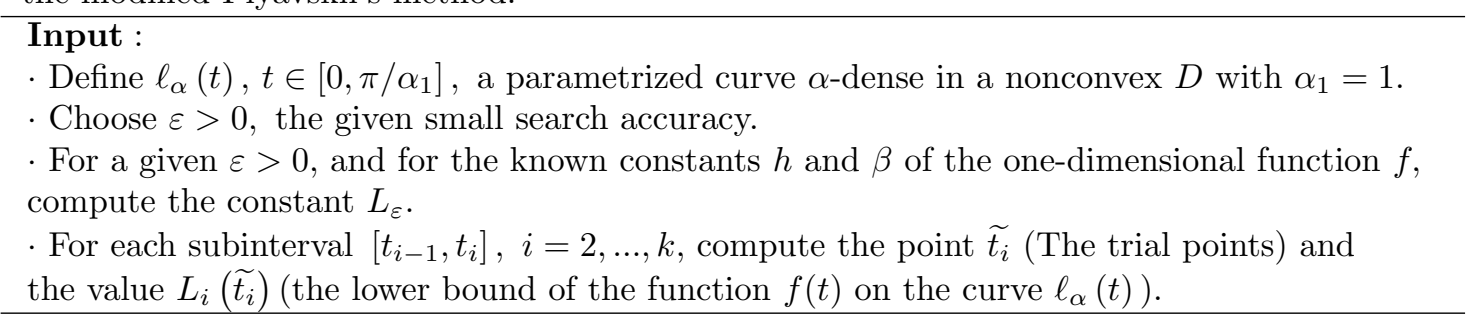




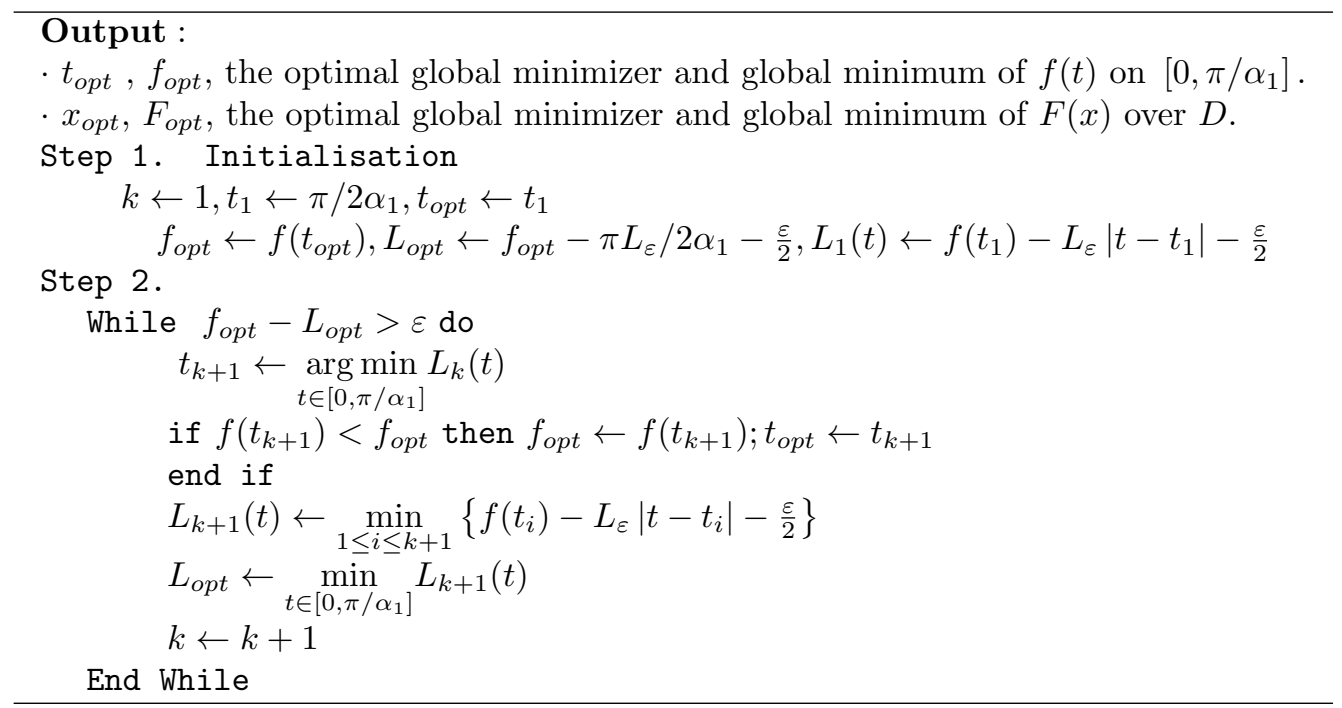

\subsection{Relationship between $\alpha$ density and $\varepsilon$ accuracy}

Theorem 5. Let $\ell_{\alpha}$ a curve $\alpha$-dense in $D$ and $\varepsilon$ an accuracy with which the global minimum of the problem (1.1) on the compact $D$ is to be searched, then we have

$$
\left\{\begin{array}{l}
\varepsilon(\alpha)=2 H\left(\delta_{n-1} \lambda_{n} \alpha\right)^{\beta} \\
\text { s.t. } \quad \varepsilon(\alpha) \rightarrow 0 .
\end{array}\right.
$$

Proof. Suppose that $x^{*}$ is an exact global minimizer of the objective function $F(x)$ on the compact $D$ and $t^{*}$ an exact global minimizer of the function $f(t)=F\left(\ell_{\alpha}(t)\right)$ on the interval $\mathbb{I}=\left[0, \pi / \alpha_{1}\right]$. Since the parameterized curve $\ell_{\alpha}(t)$ is $\delta_{n-1} \lambda_{n} \alpha$-dense in $D$, then there exists $t^{\prime} \in \mathbb{I}$ such that

$$
\left\|x^{*}-\ell_{\alpha}\left(t^{\prime}\right)\right\| \leq \delta_{n-1} \lambda_{n} \alpha \text {. }
$$

The modified algorithm of Piyavskii MPA gives us a minimizer $t^{\prime \prime}$ of the function $f(t)$ on the curve $\ell_{\alpha}(t)$ for $t \in \mathbb{I}$, satisfying

$$
\left|f\left(t^{*}\right)-f\left(t^{\prime \prime}\right)\right| \leq \frac{\varepsilon}{2}
$$

Then we have

$$
\left|F\left(x^{*}\right)-f\left(t^{\prime \prime}\right)\right| \leq \underbrace{\left|F\left(x^{*}\right)-f\left(t^{\prime}\right)\right|}_{\leq \frac{\varepsilon}{2}}+\underbrace{\left|f\left(t^{\prime}\right)-f\left(t^{\prime \prime}\right)\right|}_{\leq \frac{\varepsilon}{2}} \leq \varepsilon,
$$

and as the function $F(x)$ is Hölderian on $\Omega$, it follows that

$$
\left|F\left(x^{*}\right)-f\left(t^{\prime}\right)\right|=\left|F\left(x^{*}\right)-F\left(\ell_{\alpha}\left(t^{\prime}\right)\right)\right| \leq H\left\|x^{*}-\ell_{\alpha}\left(t^{\prime}\right)\right\|^{\beta} .
$$

So to have the desired accuracy $\varepsilon$, it suffices to choose:

$$
\alpha=\left(\frac{\varepsilon}{2 H}\right)^{1 / \beta} \frac{1}{\delta_{n-1} \lambda_{n}} .
$$

Consequently, the densification parameter $\alpha$ is calculated, depending on the required search accuracy. 


\section{Numerical examples}

In this section, we will report some numerical results of six problems using the proposed MPA algorithm for the one-dimensional problem obtained after using the dimensionality reduction method to the multivariate initial problem. The numerical results are carried out with the concrete $\alpha$-dense curves from Theorem 2. In the definition of the curves $\ell_{\alpha}(t)$, we have substituted $\alpha_{1}$ by 1 , but we can take any strictly positive value, we have also chosen these curves for their simplicity in construction. All the numerical results are implemented in MATLAB 7.1 runtime environment and the experiments have been executed at a PC with Intel Core Duo 1.6G and 1G RAM with the accuracy $\varepsilon=0.01$. Tables $1-2$ show the parameters of both the objective and the constraint functions and the computational results of optimal solution and optimal value of the six problems are summarized in Table 3. Note that the objective functions $F_{i}, 1 \leq i \leq 6$ and the constraint functions $\varphi_{i}, \psi_{i}$ of the problems are all Hölderian but neither differentiable nor Lipschitz and the feasible domains of these problems, except problems 4 and 5 , are non-convex. It should be also mentioned that the existing works concerning the multi-dimensional global optimization methods based on filling the feasible region by a curve are especially applied when the feasible region is a hyper-rectangles of $\mathbb{R}^{n}[11,19,20]$. In our approach, we have considered a category of problems when the feasible region is a compact non-convex set of $\mathbb{R}^{n}$ whose border is defined by a non-smooth functions. Although the proposed approach is applicable to a hyper-rectangles [17]. Let us note at last that the six problems are treated for the first time, due to this reason, we are unable to compare our numerical results with other works.

\section{Problem 1.}

$$
\min F_{1}\left(x_{1}, x_{2}\right)=-\left|\cos x_{1} \cos x_{2} e^{\mid 1-\frac{\left(x_{1}^{2}-x_{2}^{2}\right)^{\frac{1}{2}}}{\pi}}\right| \mid
$$

on the non-convex set $D=\left\{\left(x_{1}, x_{2}\right) \in \mathbb{R}^{2} / g_{1}\left(x_{1}, x_{2}\right) \leq 0\right.$ and $\left.g_{2}\left(x_{1}, x_{2}\right) \leq 0\right\}$ where $g_{1}\left(x_{1}, x_{2}\right)=-1+x_{2}-\frac{1}{4}\left|x_{1}\right|^{1 / 3}$ and $g_{2}\left(x_{1}, x_{2}\right)=-1-x_{2}+\frac{1}{4}\left|x_{1}\right|^{1 / 3}$ the set $D$ is contained in the rectangle $\Omega=[-1,1] \times[-1,2]$.

In this problem we take: $\varphi_{2}\left(x_{1}\right)=\frac{1}{4}\left|x_{1}\right|^{1 / 3}-1$ and $\psi_{2}\left(x_{1}\right)=\frac{1}{4}\left|x_{1}\right|^{1 / 3}+1$. The global minimizer of $F_{1}$ is $x^{*}=(0,0)^{T}$ at which both the objective and the constraint functions $\varphi_{2}\left(x_{1}\right)$ and $\psi_{2}\left(x_{1}\right)$ are Hölderian and non-smooth.

The parameterized curve $\ell_{\alpha}(t)=\left(\ell_{1}(t), \ell_{2}(t)\right)$ defined on the interval $\left[0, \pi / \alpha_{1}\right]$ by

$$
\left\{\begin{array}{l}
\ell_{1}(t)=-\cos \alpha_{1} t \\
\ell_{2}(t)=\frac{1}{4}\left|\cos \alpha_{1} t\right|^{1 / 3}-\cos \alpha_{2} t
\end{array}\right.
$$

with

$$
\left\{\begin{array}{l}
\alpha_{2} \geq \frac{2 \pi \alpha_{1}}{\alpha} \\
\text { and } \lambda_{2}=\max \left(1,1 /\left(4 \alpha^{2 / 3}\right)\right)
\end{array}\right.
$$

is $\lambda_{2} \alpha$-dense in $D$. The number $\alpha$ is calculated according to the accuracy $\varepsilon$ with which this problem is numerically solved. The problem 1 can be reduced to the following Hölder one-dimensional problem:

$$
\min _{t \in\left[0, \pi / \alpha_{1}\right]} f(t)=F_{1}\left(\ell_{\alpha}(t)\right) .
$$


Problem 2.

$\min F_{3}\left(x_{1}, x_{2}, x_{3}\right)=\left|\sin \left(0.5+9.5 \sqrt{x_{1}^{2}+x_{2}^{2}+x_{3}^{2}}\right)\right|$

subject to $\quad \sqrt{\left|x_{1}\right|}-1 \leq x_{2} \leq \sqrt{1-x_{1}^{2}}$

$\left|\sin \sqrt{x_{1}^{2}+x_{2}^{2}}\right| \leq x_{3} \leq\left|3+\cos \sqrt{x_{1}^{2}+x_{2}^{2}}\right|$

$-1 \leq x_{i} \leq 1, i=1,2$ and $0 \leq x_{3} \leq 4$

In this problem we take:

$\left\{\begin{array}{l}\varphi_{2}\left(x_{1}\right)=\sqrt{\left|x_{1}\right|}-1 \quad, \psi_{2}\left(x_{1}\right)=\sqrt{1-x_{1}^{2}} \\ \varphi_{3}\left(x_{1}, x_{2}\right)=\left|\sin \sqrt{x_{1}^{2}+x_{2}^{2}}\right|, \psi_{3}\left(x_{1}, x_{2}\right)=\left|3+\cos \sqrt{x_{1}^{2}+x_{2}^{2}}\right| .\end{array}\right.$

Then, the parameterized curve $\ell_{\alpha}(t)=\left(\ell_{1}(t), \ell_{2}(t), \ell_{3}(t)\right)$ defined on $\left[0, \pi / \alpha_{1}\right]$ by

$\left\{\begin{array}{l}\ell_{1}(t)=-\cos \alpha_{1} t \\ \ell_{2}(t)=\frac{1}{2} \varphi_{2}\left(\ell_{1}(t)\right)\left(1+\cos \alpha_{2} t\right)+\frac{1}{2} \psi_{2}\left(\ell_{1}(t)\right)\left(1-\cos \alpha_{2} t\right) \\ \ell_{3}(t)=\frac{1}{2} \varphi_{3}\left(\ell_{1}(t), \ell_{2}(t)\right)\left(1+\cos \alpha_{3} t\right)+\frac{1}{2} \psi_{3}\left(\ell_{1}(t), \ell_{2}(t)\right)\left(1-\cos \alpha_{3} t\right)\end{array}\right.$

with

$$
\left\{\begin{array}{l}
\sqrt{\alpha_{3} / \pi} \geq \frac{1}{\alpha}\left(\alpha_{2}+\alpha_{1}^{-1 / 2}+\sqrt{2} \alpha_{1}^{-2 / 3}\right) \\
\lambda_{3}=\max \left(1, \sqrt{2 / \alpha \delta_{2}}, \sqrt{3} /\left(\alpha \delta_{2}\right)^{2 / 3}\right) \\
\text { and } \delta_{2}=1+(2 / \alpha)^{0.5}
\end{array}\right.
$$

is $\delta_{2} \lambda_{3} \alpha$-dense in the feasible region.

Problem 3. Find the global minimum of non-smooth Hölder function subject to an inequality constraint.

$\min F_{2}\left(x_{1}, x_{2}\right)=\frac{1}{2} \sin \left(\sqrt{\left|x_{1}-x_{2}\right|}\right)-\frac{1}{2} \cos \left(\sqrt{\left|x_{1}+x_{2}\right|}\right)$

subject to $\quad \frac{1}{2} x_{1}^{1 / 3} \leq x_{2} \leq \exp \left(\sqrt{x_{1}}\right)$

$0 \leq x_{1} \leq 1,0 \leq x_{2} \leq 2.72$

In this problem we take $\varphi_{2}\left(x_{1}\right)=\frac{1}{2} x_{1}^{1 / 3}$ and $\psi_{2}\left(x_{1}\right)=\exp \left(\sqrt{x_{1}}\right)$.

Then, the parameterized curve $\ell_{\alpha}(t)=\left(\ell_{1}(t), \ell_{2}(t)\right)$ defined by

$\left\{\begin{array}{l}\ell_{1}(t)=\frac{1}{2}\left(1-\cos \alpha_{1} t\right) \quad \text { for } t \in\left[0, \pi / \alpha_{1}\right] \\ \ell_{2}(t)=\frac{1}{2} \varphi_{2}\left(\ell_{1}(t)\right)\left(1+\cos \alpha_{2} t\right)+\frac{1}{2} \psi_{2}\left(\ell_{1}(t)\right)\left(1-\cos \alpha_{2} t\right)\end{array}\right.$

with

$$
\left\{\begin{array}{l}
\alpha_{2} \geq \frac{2 \pi \alpha_{1}}{\alpha} \\
\text { and } \lambda_{2}=\max \left(1,1 / 2 \alpha^{2 / 3}, 2.72 / \sqrt{\alpha}\right)
\end{array}\right.
$$

is $\lambda_{2} \alpha$-dense in the feasible region.

\section{Problem 4.}

$$
\begin{aligned}
& \min F_{4}\left(x_{1}, x_{2}\right)=-20 \exp \left(-0.2 \sqrt{\frac{\left|x_{1}\right|+\left|x_{2}\right|}{2}}\right) \\
& \text { subject to } \quad x_{1}^{2}+x_{2}^{2} \leq 16 \\
& \qquad-4 \leq x_{i} \leq 4, \quad i=1,2
\end{aligned}
$$

In this problem we take:

$\left\{\begin{array}{l}a=-4, \quad b=4 \\ \varphi_{2}\left(x_{1}\right)=-\sqrt{16-x_{1}^{2}}, \psi_{2}\left(x_{1}\right)=\sqrt{16-x_{1}^{2}} .\end{array}\right.$ 
Then, the parameterized curve $\ell_{\alpha}(t)=\left(\ell_{1}(t), \ell_{2}(t)\right)$ defined by

$$
\begin{aligned}
& \left\{\begin{array}{l}
\ell_{1}(t)=-4 \cos \alpha_{1} t, \quad \text { for } \\
\ell_{2}(t)=-4 \sin \alpha_{1} t \cos \alpha_{2} t,
\end{array}\right. \\
& \text { with } \\
& \qquad\left\{\begin{array}{l}
\alpha_{2} \geq \frac{2 \pi \alpha_{1}}{\alpha} \\
\text { and } \lambda_{2}=\max (1,4 / \sqrt{\alpha})
\end{array}\right.
\end{aligned}
$$

is $\lambda_{2} \alpha$-dense in the feasible region.

\section{Problem 5.}

$\min F_{5}\left(x_{1}, x_{2}\right)=\sum_{k=1}^{3} \frac{1}{2 k}\left|\cos \left(\left(\frac{3}{2 k}+1\right) x_{1}+\frac{1}{2 k}\right)\right|\left|x_{1}-x_{2}\right|^{1 / 3}$

subject to $\quad\left(2 x_{1}-3\right)^{2}+\left(2 x_{2}-3\right)^{2}-9 \leq 0$

$$
0 \leq x_{i} \leq 3, i=1,2 \text {. }
$$

In this problem we take:

$\left\{\begin{array}{l}a=0, \quad b=3 \\ \varphi_{2}\left(x_{1}\right)=\frac{3}{2}-\sqrt{\frac{9}{4}-\left(x_{1}-\frac{3}{2}\right)^{2}}, \psi_{2}\left(x_{1}\right)=\frac{3}{2}+\sqrt{\frac{9}{4}-\left(x_{1}-\frac{3}{2}\right)^{2}} .\end{array}\right.$

Then, the parameterized curve $\ell_{\alpha}(t)=\left(\ell_{1}(t), \ell_{2}(t)\right)$ defined by

$$
\begin{aligned}
& \left\{\begin{array}{l}
\ell_{1}(t)=\frac{3}{2}\left(1-\cos \alpha_{1} t\right) \quad \text { for } t \in\left[0, \pi / \alpha_{1}\right] \\
\ell_{2}(t)=\frac{1}{2} \varphi_{2}\left(\ell_{1}(t)\right)\left(1+\cos \alpha_{2} t\right)+\frac{1}{2} \psi_{2}\left(\ell_{1}(t)\right)\left(1-\cos \alpha_{2} t\right)
\end{array}\right. \\
& \text { with } \\
& \qquad\left\{\begin{array}{l}
\alpha_{2} \geq \frac{2 \pi \alpha_{1}}{\alpha} \\
\text { and } \lambda_{2}=\max (1,3 / \sqrt{\alpha})
\end{array}\right.
\end{aligned}
$$

is $\lambda_{2} \alpha$-dense in the feasible region.

Problem 6.

$\min F_{6}\left(x_{1}, x_{2}\right)=\left|\cos \left(0.5+9.5 \sqrt{x_{1}^{2}+x_{2}^{2}}\right)\right|$

subject to $\quad\left|x_{1}\right|-\left(x_{2}+1\right)^{2} \leq 0$

$$
x_{1}^{2}+x_{1}^{2} \leq 1
$$

The objective function $F_{6}$ has 0 as global minimum value and has a continuum of global minimizers on its non-convex feasible region. In this problem we take

$\left\{\begin{array}{l}a=-1, \quad b=1 \\ \varphi_{2}\left(x_{1}\right)=\sqrt{\left|x_{1}\right|}-1, \psi_{2}\left(x_{1}\right)=\sqrt{1-x_{1}^{2}} .\end{array}\right.$

Then, the parameterized curve $\ell_{\alpha}(t)=\left(\ell_{1}(t), \ell_{2}(t)\right)$ defined by

$$
\left\{\begin{array}{l}
\ell_{1}(t)=-\cos \alpha_{1} t \quad \text { for } t \in\left[0, \pi / \alpha_{1}\right] \\
\ell_{2}(t)=\left(-1+\sin \alpha_{1} t+\sqrt{\left|-\cos \alpha_{1} t\right|}\right) \cos ^{2} \frac{\alpha_{2} t}{2}+\sin \alpha_{1} t,
\end{array}\right.
$$

with

$$
\left\{\begin{array}{l}
\alpha_{2} \geq \frac{2 \pi \alpha_{1}}{\alpha} \\
\text { and } \lambda_{2}=\max (1, \sqrt{2 / \alpha}),
\end{array}\right.
$$

is $\lambda_{2} \alpha$-dense in the feasible region. 


\begin{tabular}{cccc}
\hline Problem No. & Hölder constant, Hölder exponent & Region $\Omega$ & Source of $F_{i}(x)$ \\
\cline { 2 - 3 }$F_{i}(x)$ & $(5.0679,0.5)$ & {$[-1,1] \times[-1,2]$} & {$[14]$} \\
2 & $(13.43,0.5)$ & {$[-1,1]^{2} \times[0,4]$} & {$[3]$} \\
3 & $(1,0.5)$ & {$[0,1] \times[0,2.71]$} & {$[19]$} \\
4 & $(154.43,0.5)$ & {$[-4,4]^{2}$} & {$[14]$} \\
5 & $(15.8,1 / 3)$ & {$[0,3]^{2}$} & {$[19]$} \\
6 & $(13.43,0.5)$ & {$[-1,1]^{2}$} & {$[3]$} \\
\hline
\end{tabular}

Table 1: The parameters of the non-smooth Hölder objective functions

\begin{tabular}{cccccc}
\hline \multirow{2}{*}{ Problem No. } & \multicolumn{3}{c}{ Hölder constant, Hölder exponent } & \multirow{2}{*}{ Region $\Omega_{i}$} \\
\cline { 2 - 5 } & $\varphi_{2}\left(x_{1}\right)$ & $\psi_{2}\left(x_{1}\right)$ & $\varphi_{3}\left(x_{1}, x_{2}\right)$ & $\psi_{3}\left(x_{1}, x_{2}\right)$ & \\
\hline 1 & $(0.25,1 / 3)$ & $(0.25,1 / 3)$ & $\cdot$ & $\cdot$ & {$[-1,1]$} \\
2 & $(1,0.5)$ & $(\sqrt{2}, 0.5)$ & $(\sqrt{2}, 0.5)$ & $(\sqrt{2}, 0.5)$ & {$[-1,1],[-1,1]^{2}$} \\
3 & $(0.5,1 / 3)$ & $(2.72,0.5)$ &. &. & {$[0,1]$} \\
4 & $(4,0.5)$ & $(4,0.5)$ &. &. & {$[-4,4]$} \\
5 & $(\sqrt{3}, 0.5)$ & $(\sqrt{3}, 0.5)$ &. &. & {$[0,3]$} \\
6 & $(1,0.5)$ & $(\sqrt{2}, 0.5)$ &. &. & {$[-1,1]$} \\
\hline
\end{tabular}

Table 2: The parameters of the non-smooth Hölder constraint functions

\begin{tabular}{ccccc}
\hline Problem No. & $\alpha$ & $\alpha_{1}$ & $x_{\text {opt }}$ & $F_{\text {opt }}$ \\
\hline 1 & 0.2 & 1 & $(-0.00012,-0.00231)$ & -2.7093 \\
2 & 0.15 & 1 & $(0.0010,0.0004,0.0012)$ & 0.47943 \\
3 & 0.3 & 1 & $(0.0178,0.1206)$ & -0.4893 \\
4 & 0.15 & 1 & $(0.0011,0.0002)$ & -19.9571 \\
5 & 0.2 & 1 & $(1.4753,1.4945)$ & 0.02439 \\
6 & 0.2 & 1 & $(0.00234,0.000167)$ & 0.87758 \\
\hline
\end{tabular}

Table 3: Computational results of problems 1-6 using DR-MPA 
The symbols used in Table 3 are given as follows: $\alpha$ and $\alpha_{1}$ are the strictly positive parameters of the densification of the curve $\ell_{\alpha}(t)$ on $\left[0, \pi / \alpha_{1}\right], x_{o p t}$ is the optimal global minimizer of $F(x)$ on $D$ and $F_{\text {opt }}$ is the optimal global minimum value of $F(x)$ on $D$.

\section{Conclusion}

A new coupled method is proposed in this paper, called DR-MPA method, for solving nonconvex, non-smooth constrained global optimization problem. We have considered a class of problems when the objective and the constraint functions are all Hölderian and non-differentiable, and we have shown how to solve such problems without using the Lagrangian and the dual problem. Indeed, this class of problems is very difficult to treat, because the non-differentiability of the objective and the constraint functions, with the existence of a large number of local and global extrema. This method is a combination of two methods. In fact, by means of the dimensionality reduction method, we converted the multivariate initial problem to a univariate Hölder non-differentiable one.

Before starting the minimization procedure, we approach the non-convex feasible area with an $\alpha$-dense curve by constructing explicitly a parametric representation $x_{i}=\ell_{i}(t), 1 \leq i \leq n$ defined on the interval $\mathbb{I}$ of $\mathbb{R}$. For minimizing the univariate Hölder function on the interval $\mathbb{I}$, we have proposed a modification of Piyavskii's algorithm MPA which we have introduced in [17] and which does not require any other assumptions but only the knowledge of the constants $h$ and $\beta$. We have used the Piyavskii's algorithm since it is the best known and most studied in the literature.

The dimensionality reduction method is rather effective for dealing with difficult problems and its numerical implementation is very simple and does not require a lot of storage space. Although this method has its own advantages, the disadvantage is that it generates an excessive number of evaluation points of the objective function, particularly when the dimension of the feasible set is greater than four or when the objective function is subject to great variations. It wold also be interesting to test the algorithms on a larger number of test problems and compare with other algorithms.

\section{References}

[1] Ammar, H. and Cherruault, Y. (1993). Approximation of a several variables function by a one variable function and application to global optimization. Mathematical and Computer Modelling, 18(2), 17-21. doi: 10.1016/0895-7177(93)90003-h

[2] Butz, A. R. (1968). Space filling curves and mathematical programming. Information and control, 12(4), 313-330. doi: 10.1016/s0019-9958(68)90367-7

[3] Delgado, P. M. and Galperin, E. A. (2006). Global optimization over general compact sets by the beta algorithm: A MAPLE Code. Computers and Mathematics with Applications, 52(1-2), 33-54. doi: 10.1016/j.camwa.2006.08.003

[4] Gourdin, E., Jaumard, B. and Ellaia, R. (1996). Global Optimization of Hölder function. Journal of Global Optimization, 8(4), 323-348. doi: 10.1007/bf02403997

[5] Guettal, D. and Ziadi, A. (2012). Reducing Transformation and Global Optimization. Applied Mathematics and Computation, 218(10), 5848-5860. doi: 10.1016/j.amc.2011.11.053

[6] Hanjoul, P., Hansen, P., Peeters, D. and Thisse, J. F. (1990). Uncapacitated plant location under alternative space price policies. Management Science, 36(1), 41-57. doi: 10.1287/mnsc.36.1.41

[7] Horst, R. and Pardalos, P. M. (1995). Handbook of Global Optimization. Nonconvex Optimization and Its Applications, 2. Springer-Verlag, Boston. doi: 10.1007/978-1-4615-2025-2

[8] Horst, R. and Tuy, H. (1993). Global Optimization, Deterministic Approaches. Springer-Verlag, Berlin. doi: 10.1007/978-3-662-03199-5 
[9] Kiatsupaibul, S., Smith, L. R. and Zabinsky, Z. B. (2016). Solving infinite horizon optimization problems through analysis of a one-dimensional global optimization problem. Journal of Global Optimization, 66(4), 711-727. doi: 10.1007/s10898-016-0423-7

[10] Lera, D. and Sergeyev, Y. D. (2002). Global Minimization Algorithms for Hölder Functions BIT Numerical Mathematics, 42(1), 119-133. doi: 10.1023/a:1021926320198

[11] Lera, D. and Sergeyev, Y. D. (2010). Lipschitz and Hölder global optimization using space-filling curves. Applied Numerical Mathematics, 60(1-2), 115-129. doi: 10.1016/j.apnum.2009.10.004

[12] Hou, Z. E. and Duan, F. J. (2007). The approximation algorithm for solving a sort of non-smooth programming. Applied Mathematics and Computation, 186(2), 1511-1519. doi: 10.1016/j.amc.2006.06.129

[13] Mishra, S. H. (2006). Some new test functions for global optimization and performance of repulsive particle swarm method. Available at SSRN, 1-12. doi: 10.2139/ssrn.926132

[14] Mora, G. and Cherruault, Y. (1997). Characterization and generation of $\alpha$-dense curves. Computers and Mathematics with Applications, 33(9), 83-91. doi: 10.1016/s0898-1221(97)00067-9

[15] Pinter, J. D. (1996). Global Optimization in Action, Continuous and Lipschitz Optimization: Algorithm, Implementations and Applications. Book Series of the Nonconvex Optimization and Its Application. Kluwer Academic Publisher, Dordrecht. Springer: New York. doi: 10.1007/978-14757-2502-5

[16] Piyavskii, S. A. (1972). An algorithm for finding the absolute extremum of a function. USSR Computational Mathematics and Mathematical Physics, 12(4), 57-67. doi: 10.1016/00415553(72)90115-2

[17] Rahal, M. and Ziadi, A. (2008). A new extension of Piyavskii's method to Hölder functions of several variables. Applied Mathematics and Computation, 197(2), 478-488. doi: 10.1016/j.amc.2007.07.067

[18] Sagan, H. (1994). Space-filling curves. Springer-Verlag, New York. doi: 10.1007/978-1-4612-0871-6

[19] Sergeyev, Y. D., Strongin, R. G. and Lera, D. (2013). Introduction to Global Optimization Exploiting Space-Filling-Curves. Springer Briefs in Optimization. Springer, New York. doi: 10.1007/9781-4614-8042-6

[20] Strongin, R. G. (1992). Algorithms for multi-extremal programming problems employing the set of joint space-filling curves. Journal of Global Optimization, 2(4), 357-378. doi: 10.1007/bf00122428

[21] Törn, A. and Žilinska, A. (1989). Global optimization. Lecture Notes in Computer Sciences, 350. Springer-Verlag. https://doi.org/10.1007/3-540-50871-6

[22] Vanderbei, R. J. (1999). Extension of Piyavskii's algorithm to continuous global optimization. Journal of Global Optimization, 14(2), 205-216. doi: 10.1023/a:1008395413111

[23] Ziadi, A. and Cherruault, Y. (1998). Generation of $\alpha$-dense curves in a cube of $\mathbb{R}^{n}$. Kybernetes, 27(4), 416-425. doi: 10.1108/eum0000000004524

[24] Ziadi, A. and Cherruault, Y. (2000). Generation of $\alpha$-dense curves and application to global optimization. Kybernetes, 29(1), 71-82. doi: 10.1108/03684920010308871

[25] Ziadi, A., Cherruault, Y. and Mora, G.(2000). The existence of $\alpha$-dense curves with minimal lenght in a metric space. Kybernetes, 29(2), 219-230. doi: 10.1108/03684920010312803

[26] Ziadi, A., Cherruault, Y. and Mora, G. (2001). Global optimization: A new variant of the Alienor method. Computers and Mathematics with Applications, 41(1-2), 63-71. doi: 10.1016/S08981221(01)85006-9

[27] Ziadi, R., Bencherif-Madani, A. and Ellaia, R. (2016). Continuous global optimization through the generation of parametric curves. Applied Mathematics and Computation, 282, 65-83. doi: 10.1016/j.amc.2016.01.067 